\title{
TEST VLPC CRYOSTAT: \\ PROGRAMMING AND CONFIGURATION OF CRYOGENIC AND TEMPERATURE INSTRUMENTS
}

DO ENGINEERING NOTE: 3823.113-EN-470

AUGUST 11,1997

MARIUSZ ZACZEK

DO - MECHANICAL GROUP

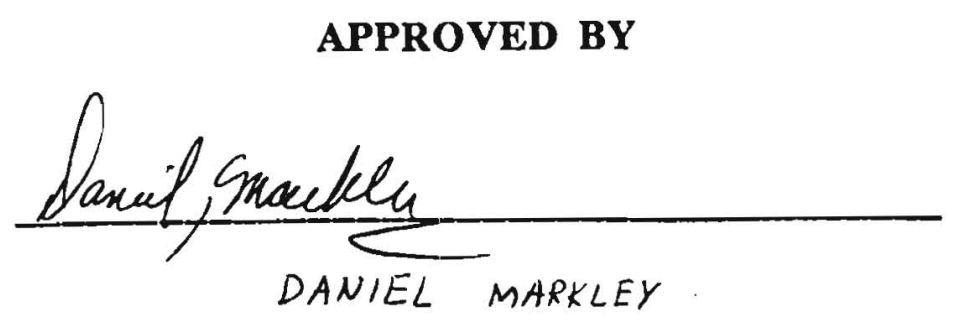




\section{Figure and Tables}

Figure or Table

1. Figure 1: Schematic of PLC and Modules

2. Figure 2: Wiring Diagram of an RTD

3. Figure 3: TE306 Special Function Ladder Logic Rung

4. Figure 4: LOOP-A Auto/Man Control Ladder Logic Rung

5. Figure 5. Valve FCV-312-HE Ladder Logic

6. Figure 6. (LVF) Loop V-Flags

7. Figure 7. Loop \#1 Ladder Logic Flow Diagram
Page \#

2

4

5

7

8

8

9 


\section{Introduction}

Currently, Fermi National Accelerator Laboratory is in the midst of a site wide upgrade. Here at D-Zero, one of the components of this upgrade will be the construction of two 48 cassette VLPC cryostats which will be the heart of the new magnetic central fiber tracker. A VLPC or Visible Light Photon Counter is a device that allows physicists to more accurately calculate the origins of particles ejected during a proton - anti-proton collision in the detector. Inside the detector is an optical fiber barrel which surrounds the collision point. When an ejected particle strikes a fiber the result is the release of photons which travel along the fiber until they reach the bottom of a cassette hitting the VLPC chip. These impacts result in voltages which are read by the VLPC chips and sent to the computer for future analysis. From these voltages, physicists can determine the origins of the particles, their charges, their speeds and other information as well.

Within the last few months a test VLPC has been built at D-Zero. This VLPC is comprised of four rectangular cassettes each equipped with heating elements, RTDs and 1024 VLPC chips. This particular configuration is unique here at Fermilab. Other VLPC cryostats that have been built were usually cylindrical in their geometry. The purpose of this test VLPC is to have on hand a reliable model to aid in understanding the complexities involved in running such devices at approximately $10 \mathrm{~K}$, as well as to prepare for the eventual 48 cassette VLPCs to come. In particular, an important aspect of this test phase is to check the engineering involved so that the final cryostats are designed correctly. Also, this test cryostat will enable us to check each of the cassettes to be used in the final cryostat and correct any problems before hand.

\section{Purpose}

This engineering note has been written in order to describe the setup of the VLPC as it pertains to the programming, configuration and control of all of the cryogenic and temperature instruments. These instruments include the two pneumatic valves, the pressure transmitter, heating elements of the cassettes, and the various RTDs scattered throughout.

(Note: Please refer to the 'Test VLPC: Programming and Control of the Cryogenic and Temperature Instruments" manual for all the RTD curve-fits, special functions, ladder logic, flow diagrams, etc. which will be discussed throughout this engineering note.)

\section{Computer Configuration}

The programming and control of these instruments was done using a SIEMENS 545 Programmable Logic Controller (PLC) along with a Gateway 2000 - Pentium 60 
computer (user interface) running TISOFT (used for programming) and FIX DMACS (used for graphical display and control) software. In Figure 1 below, we can see a schematic of PLC including the input/output signal conditioning modules. (Notice the two 8 Channel Analog Output modules require an external 24 VDC power source to be fully operational.)

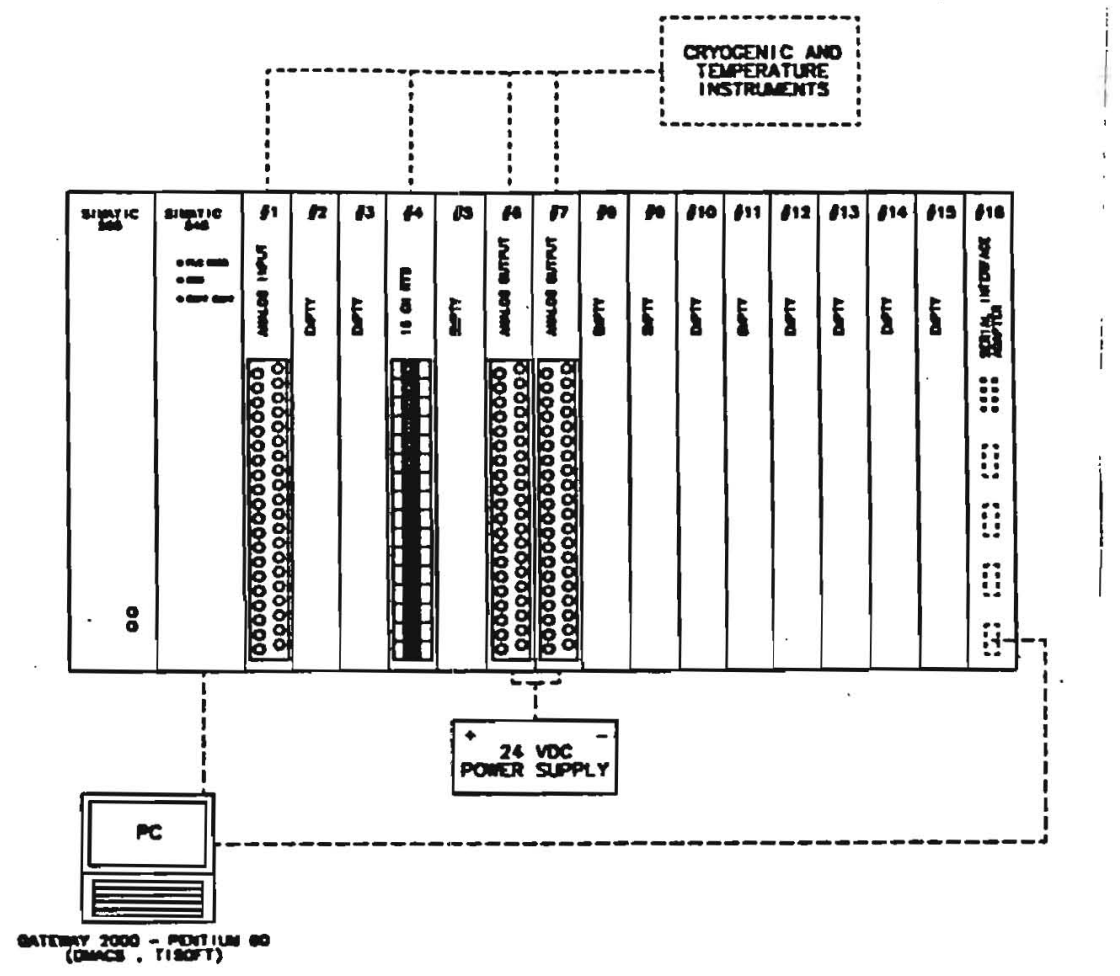

Figure 1: Schematic of the SIEMENS 545 PLC and modules

The cryogenic and temperature instruments of the test VLPC are individually connected to the appropriate modules of the PLC. A wiring diagram of each module's instruments has been provided in 'Test VLPC: Programming and Control of the Cryogenic and Temperature Instruments" manual. The PLC is connected via two serial cables to the Gateway 2000 - Pentium 60 computer. One serial cable is used to transmit and receive data between the PLC and the DMACS software. The other serial cable is used by TISOFT for programming of the PLC itself. As mentioned previously, the DMACS software is an easy to use graphical interface for the operator. The on-screen display allows the operator to view the various system components, display instrument data in real time as well as control the instruments via the computer. TISOFT is a commercially available PLC programming software developed by Texas Instruments. The version of TISOFT used for this test setup is a simple ASCI graphical display. A newer version know as SOFTSHOP is currently available but is not used here since I am more familiar and proficient in the use of TISOFT. 


\section{Module Configuration}

\section{Slot \#1}

In Figure 1 we see that slot 1 of the PLC crate is occupied by an 8 ch Analog Input Module [SIEMENS Simatic 505-6108A]. The signal conditioning of this module assigns 8 WX (analog input) words. These occupy memory locations WX1 through WX8. At this time only WX1 is being used. It is connected to the test VLPC vacuum transmitter (PT$619-\mathrm{HE}$ ) having a range of $0-25$ psig.

(Note: An table containing the configuration of each module as well as the assignment of the words has been provided in the I/O Map section of this Engineering Note.)

\section{Slots \#2 and \#3}

Slots 2 and 3 are currently left empty but can be filled and configured at anytime. To install a module, simply disconnect the PLC from the power supply, insert the module in the desired slot and configure the I/O of the module using the TISOFT program. TISOFT can be run using the RUN command line in the start menu of the Gateway 2000 computer. Simply type in (C:ITNTI565.BAT P2). Once the program has reached the main TISOFT screen, go ONLINE [F4] and configure the I/O [SPACE , F3] followed by CONFIG [F2].

\section{Slot \#4}

Slot 4 contains the $16 \mathrm{pt}$ RTD input module [CTI 2557]. This modules reads individual resistances of 4 wire resistors which are then converted to temperature using special function programs within the PLC. The module has assigned to it $16 \mathrm{WX}$ words ranging from WX25 to WX41. Words WX25 through WX28 are assigned to RTDs TE200, TE202, TE204 and TE206, respectively. These four RTDs are currently unavailable and there status is yet to be determined. One possibility is that four of the other RTDs will be moved to these locations while another possibility is that completely new RTDs will be installed. Words WX29 through WX39 are occupied by eleven RTDs: three on the VLPC and eight on the cassettes .

\section{Slot \#5}

Slot 5 is left empty. (see slots $2 \& 3$ )

\section{Slots \#6 \& \#7}

These two slots are each occupied by an $8 \mathrm{ch}$ Analog Output module [SIEMENS Simatic 505-6208A]. Also, each of these modules is connected to an external 24 VDC power supply in order to be fully operational. The modules are assigned 8 WY (analog output) words each, with slot 6 configured for WY49 through WY56 while slot 7 is configured for WY57 through WY64. The output of each cassette's heater is controlled by 
words WY49, WY50, WY51 and WY52 for heaters on cassette one through four, respectively. These output values are calculated using loop-mimicking special function programs which employ PI (Proportional and Integral) control of the loops. WY53 and WY54 control the loop output for the two pneumatic valves. This output is simply the percentage of the valve position e.g. $0.0 \%$ signifies a closed valve while $100.0 \%$ signifies a fully open valve. The valves are controlled both by loops as well as by special function programs; they will be discussed completely in an upcoming section.

Slots \#8 - \#15

Slots 8 through 15 are left empty. (see slots 2 \& 3)

\section{Slot \#16}

Slot 16 is the Serial Interface Adapter. It serves as the main link between the PLC and the user interface (Gateway 2000 computer). The module is configured with six WY and two WX words, which are used by the module to transmit and receive data.

\section{Programming of RTDs Special Functions}

As mentioned previously, the RTDs (Resistance - Temperature Devices) are connected to the 16 ch RTD module of the PLC crate. These RTDs are simple 4 wire resistors shown schematically below.

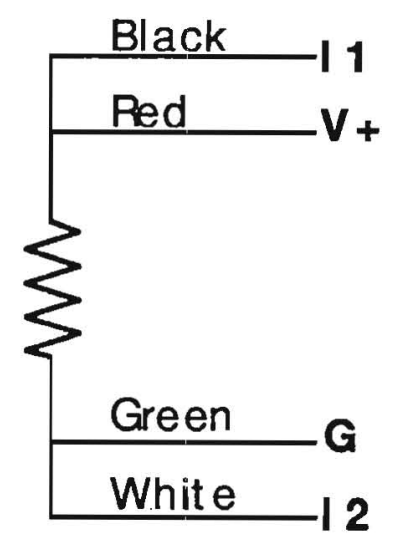

\section{Figure 2: Wiring Diagram of an RTD}

The black and white wires carry the current across the resistor whole the red and green wires measure the actual resistance across it. The red and green wires are connected to the actual resistor as closely as possible in order to avoid counting the resistance of the wires leading to the resistor itself. The resistance read by the module (slot 4) ranges from 0 to 1000 ohms. This value is transferred as an integer to the PLC via a WX integer ranging from 0 to 32000 . The PLC then converts this integer to the appropriate resistance which in turn is used in an equation to calculate the corresponding temperature in Kelvin. The 
equations have been obtained using the Microsoft EXCEL spreadsheet program by curvefitting the given calibration data received from the manufacturer. Each RTD has a unique set of calibration data and so each RTD requires a separate special function be written in order to convert the resistance to a temperature. Most of the time the data must be broken down into separate curves to gain the higher accuracy required. The equations obtained for each of the RTDs listed in this note have an error on the order of $+/-0.002 \mathrm{~K}$ in the low temperature range $(3-20 \mathrm{~K})$, while in the higher range $(20-300 \mathrm{~K})$ the error may increase to $+/-1.0 \mathrm{~K}$. As mentioned previously, the equations for each RTD are combined into a special function program. As an example let us look at the RTD - TE306 -- The special function program and curve-fit data for this RTD are provided in the appendix of this engineering note as is a table of all V-memory; all of the RTDs are available in the Test VLPC manual mentioned previously. This particular RTD has been curve fit using two curves, one for the 3-24 $\mathrm{K}$ range and the other for the $24-300 \mathrm{~K}$ range. The first IF-ELSEENDIF logic operation determines if the analog input word (WX30) is reading a negative value and acts accordingly to convert the word to a real positive value (V11. = resistance [ $\sim 0-1060$ ohms]). Next, the program determines in which range the above calculated resistance is located. It then uses the appropriate equation to convert the resistance to a temperature (V43. in Kelvin). The UNSCALE command at the bottom of the special function is unique only to TE306 and TE310, and is used to help control the valve loops which use these RTDs as their process variables. In order for the above calculations to be performed, the special function itself must me initialized. This is done through simple ladder logic programming as shown below.

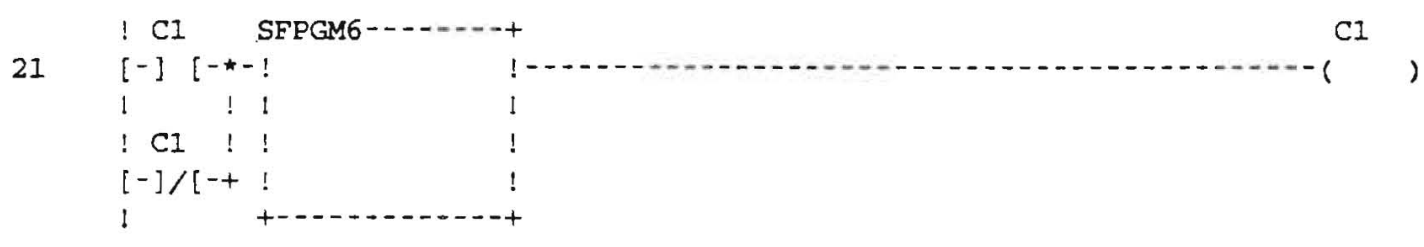

Figure 3. TE306 Special Function Ladder Logic Rung

The above ladder "rung" is characteristic of each RTD special function. The open and closed C1 contacts ensure that SFPGM6 is run every time the PLC goes through the ladder. 


\section{Programming of Heaters}

Each of the four cassettes of the test VLPC contain two heaters which are used to warm up the cassette based on the temperature readings received from the RTDs on that cassette. Although there are two heaters, they will be controlled as one with their output originating from one analog output word (WY). A PI ( Proportional and Integral ) control has been selected for the heaters and so a loop will need to built in order to achieve this. Because the current VLPC setup is only temporary it would be unwise to use a standard SCADA node. Instead a direct driver control will be used. The TID driver ( Advanced Texas Instruments Direct I/O Driver) provides the software interface and communications protocol between FIX DMACS and FIX MMI software and the PLC. The heaters require the use of a loop to control them, but since the number of loops in the PLC is limited to 64, and in the future VLPCs the number of heaters may grow to 98 or more, another method of loop control was necessary. The solution was to build a loop-mimicking special function program. This program would perform the necessary PI calculations using V-memory in place of the commonly used LVFs, LMNs, LSPs,etc. The V-memory is used because the TID driver allows only certain memory types to be transferred to DMACS from the PLC and LVF, LMN, etc. are not included. The necessary PID algorithm has been obtained from the Siemens Programming_Reference Manual Section 9.3, page 9-6. The special function program for a heater loop would calculate the output of the loop using temperature data as well, and any gains that the user may have specified through the DMACS heater control pages, and send the results to DMACS as a variable float (VF) value.

As a example, let us analyze the special function for the heater in cassette number one. This special function program is named LOOP-A and corresponds to SFPGM16 (see the appendix for a copy of the program). One of the main controls of the heater loop is the ability of the operator to set the loop to Manual or Automatic. The operator simply hits the MANUAL or AUTOMATIC push buttons on his display which in turn control ladder logic contact C2 - see Figure 4 below. (Note: Contact C2 corresponds to LOOP-A, the other heaters use different contacts. A listing of all the relays used for this test VLPC is included in the appendix.) 


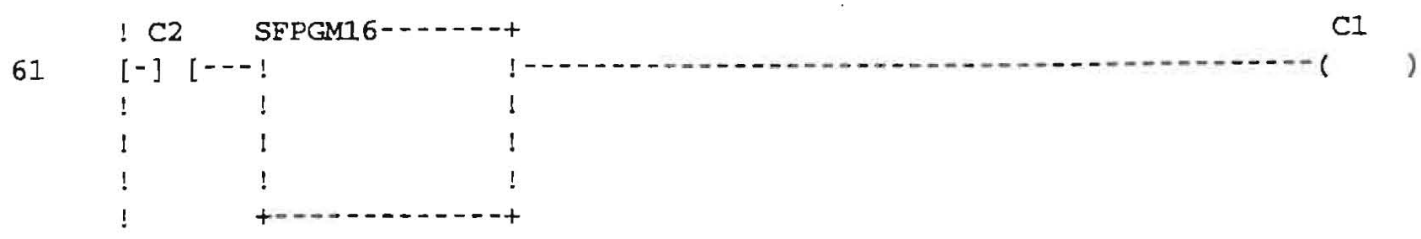

\section{Figure 4: LOOP-A Auto/Man Control Ladder Logic Rung}

If the loop is placed in manual mode, the operator can control the heater output directly. The DMACS picture for heater \#1 allows the user to double-click on the output data and select the value with the following provision: 0.0 represents an output of $0.0 \%$ while 1.0 represents an output of $100.0 \%$. The $0-100 \%$ corresponds to the amount of current [ $0.0-20.0 \mathrm{~mA}$ ] that should be transferred via the output modules (slots \#6 and \#7) to the heaters.

On the other hand, if the loop is in the automatic mode, the user controls the heater output by manipulating the gains of the loop along with the desired set-point. The set-point corresponds to the desired temperature of the cassettes with the process variable being an RTD located at the cassette base.

The LOOP-A special function program has been programmed in the following manner. First the heater output (WY49) is scaled to a real output (n-1) represented by V111. If the incoming output (n-1) is overrange or underrange it is then initialized to 1.0 or 0.0 , respectively, otherwise it is left unaltered. The gains and sample rates are read in next and the error and bias are calculated. The output is calculated next and the result is scaled into an analog output integer (WY49) - notice this output (n) will be the (n-1) output when the next cycle of the loop begins. Finally, the output signal is transferred to the heaters by means of the analog output modules and the heaters then respond accordingly. The special function is executed every time the PLC runs through the ladder.

\section{Programming of Valves}

The two pneumatic valves of the VLPC are similar to the heaters in that they too need a loop to control their output. But as before, only certain memory types are transferable between the PLC and DMACS when the TID driver is used. Therefore, to achieve accurate control of the valves a standard loop has been assigned for each valve. In addition to these loops, special function programs have also been written in order to transfer data between the PLC and DMACS through the TID driver (see the appendix for 
copies of the loops and special function programs. The ladder logic associated with valve FCV-312-HE is shown in Figure 5 below.

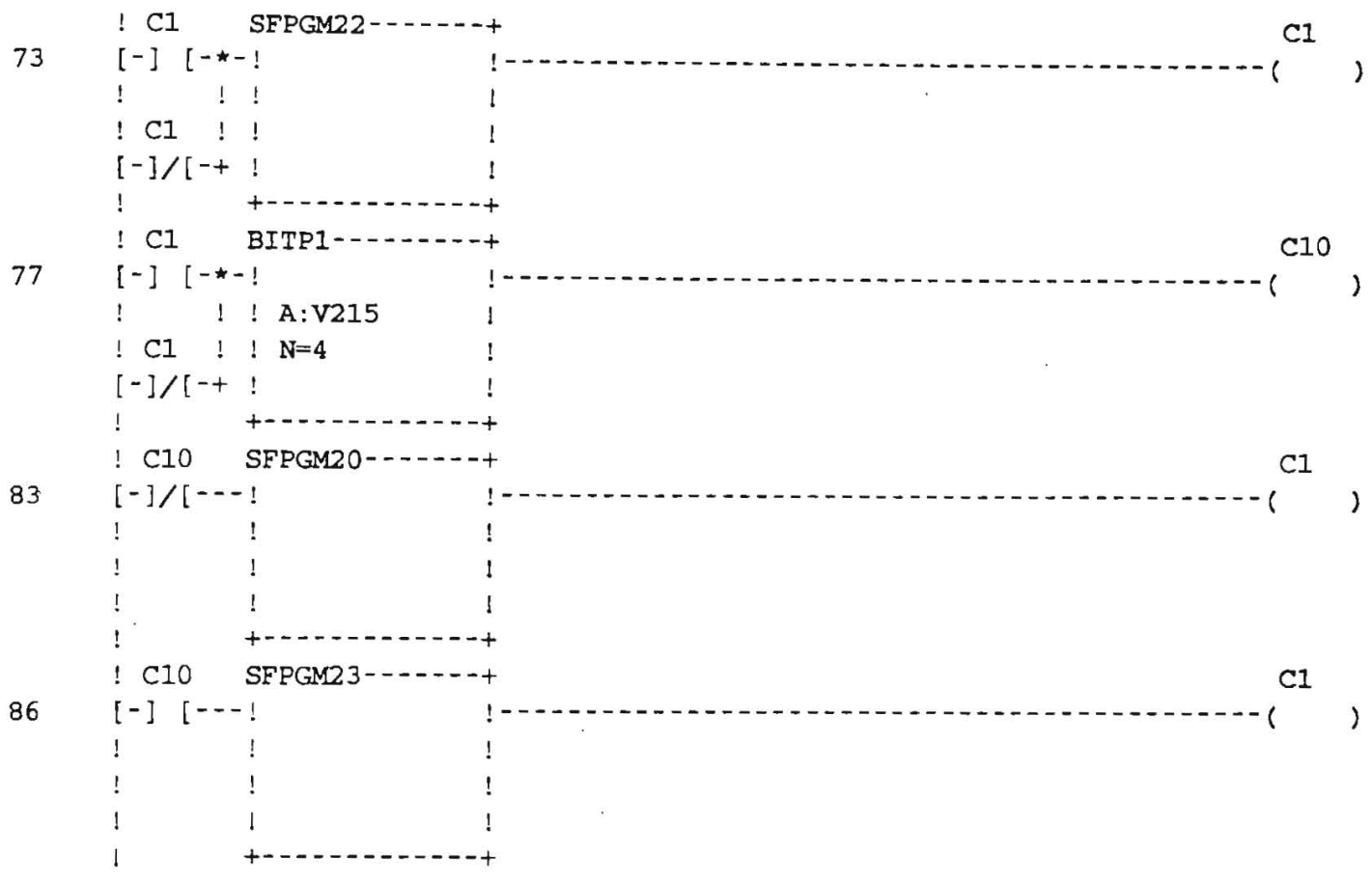

\section{Figure 5. Valve FCV-312-HE Ladder Logic}

As in the heaters, the valve must be able to be in automatic or manual modes. A loop V-flag variable (LVF) is used to instruct the loop to achieve the desired mode. By manipulating certain bits with the LVF word, the operator can set mode of the loop accordingly. The binary word (LVF1) associated with loop \#1 contains 16 bits, with the leftmost being bit-15 while the rightmost one is bit- 0 .

$$
\begin{gathered}
{[0|0| 0|0| 0|0| 0|0| 0|0| 0|0| 0|0| 0 \mid 0]} \\
\text { bit-15 bit-0 }
\end{gathered}
$$

\begin{tabular}{|c|c|c|}
\hline BIT & VALUE & ACTION \\
\hline 15 & 1 & Set loop to Manual \\
\hline 14 & 1 & Set loop to Auto \\
\hline 12 & 1 & Loop status: in Auto \\
\hline 12 & 0 & Loop status: in Manual \\
\hline
\end{tabular}

Figure 6. (LVF) Loop V-Flags 
SFPGM22 is used to copy the control V-memory to the appropriate LVFs. For the case of valve FCV-312-HE this means continuously copying V201 to LVF1. This allows the user to change V201 through DMACS and transfer this value to the PLC through the TID driver where it is copied to LVF1 and the loop acts according. The output of the loop is controlled using ladder logic. In ladder rung 77 of Figure 5 we see the box BITP1 which reads the fourth bit from the left $(\mathrm{N}=4)$-- corresponding to bit-12 -- of V201 and turns coil C10 on if the value is 1. This in turn will execute SFPGM23 (Manual Mode) and disable SFPGM20 (Automatic Mode). On the other hand, if the bit-12 is 0 the reverse will occur. A flow diagram of the logic is displayed below.

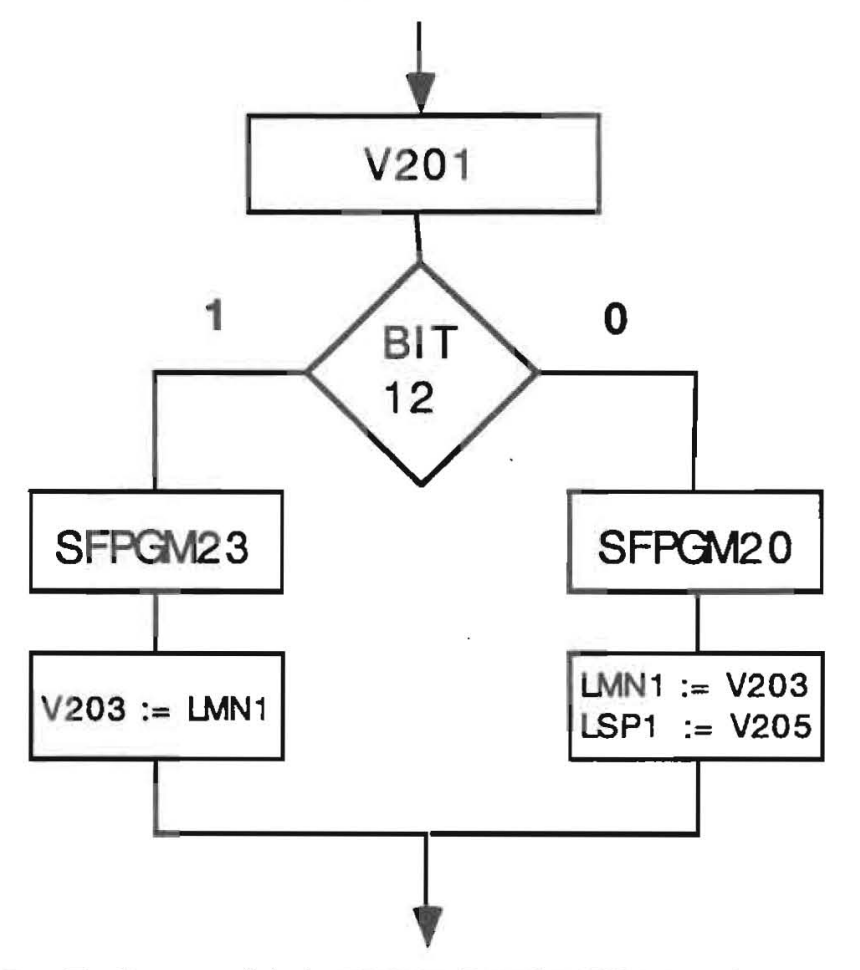

Figure 7. Loop \#1 Ladder Logic Flow Diagram

The operator can set the loop mode by hitting the A/M toggle push-button of the DMACS valve picture (testpod.odf). The action above will result in one of the following situations:

If the loop is already in the automatic mode (i.e. bit-12 is 1) then the hitting the button will set bit 15 in V201 (LVF1) to 1 using the DMACS database block LOOP1MAN (Note: The command in the database block is: D11:V201:15). After a few seconds this bit is cleared back to 0 and the loop will be in manual mode. The status of the loop can be determined by looking at bit 12 of V201. When in manual mode the output and set point 
are controlled via SFPGM20, which had been activated in the ladder, by the operator. The operator sets the loop output (LMN1) by modifying V203 while the set point (LSP1) is determined when the operator modifies V205. The set point will not be update in the loop until the loop is set to automatic mode; in the mean time it remains in V-memory V205.

If the loop is in manual mode (i.e. bit-12 is 0 ) then the DMACS database block (LOOP1AUT) will set bit 14 to 1 (D11:V201:14 in the DMACS database block). Again, after a few seconds this bit will also be reset to 0 while bit-12 of V201 should now be reading 1). In automatic mode SFPGM23 is the active program which is used to obtain the output (LMN1) from the loop and copy it to a V-memory (V203) and send it to DMACS to be displayed. This special function program is nothing more than a status program, modifying V203 externally has no affect on the loop output. On the other hand, modifying the set point (V205) has a direct affect on the loop since the set point value is being continuously updated by the loop.

The loop for valve FCV-308-HE acts in exactly the same manner but it is controlled via a different set of V-memory values. As mentioned previously, a list of all V-memory and Relays has been provided in the appendix of this engineering note, as are all of the special functions and ladder logic that has been used.

\section{DMACS}

The graphical interface to be used by the operator is know as FIX DMACS. DMACS is a commercially available program which serves as a graphical user interface for controlling various devices. The user can draw two-dimensional representations of valves, pumps, RTDs, etc. with output readings and control these objects and the data by simply pointing and clicking on them with a mouse. (Note: See the appendix for copies of the pictures.) The first drawing associated with this test VLPC is called testvlpc.odf and is located in the C:IWDMACS PICSDO directory. This picture is a more graphical drawing of the flow diagram for the VLPC. Using this picture, the user can view the temperature associated with the various RTDs, the cryostat vacuum pressure, the heater and valve modes as well as control the valves. The four cassettes as well as the two valves are each clickable and bring up separate pictures to control them. The valve picture testpod.odf allows the user to change modes as well as modifying valve position (only in manual mode) and changing the set point (both in manual and automatic modes). In the heater pictures (casX.odf where $\mathrm{X}$ is $1,2,3$ or 4 representing the four cassettes), both the resistance as well the temperature of the RTD is displayed. Also, the user can change the heater loop modes and control the output either directly (in manual mode only) or via the proportional and integral gains. 


\section{Trouble-Shooting}

\begin{tabular}{|c|c|}
\hline Problem & Possible Solutions \\
\hline $\begin{array}{l}\text { Can't change valve or heater to manual or } \\
\text { automatic modes }\end{array}$ & $\begin{array}{l}\text { ** Check that the PLC is in RUN mode } \\
\text { [RUN led is on] } \\
\text { ** Using TISOFT, enter chart mode and } \\
\text { using CHANGVAL-[F2] set } \\
\begin{array}{lll}\text { FCV-312-HE } & \text { V201 }=0 & \text { [F8] } \\
\text { FCV-308-HE } & \text { V207 }=0 & \text { [F8] }\end{array}\end{array}$ \\
\hline $\begin{array}{l}\text { Valves don't seem to be responding to } \\
\text { commands (they don't physically move) }\end{array}$ & 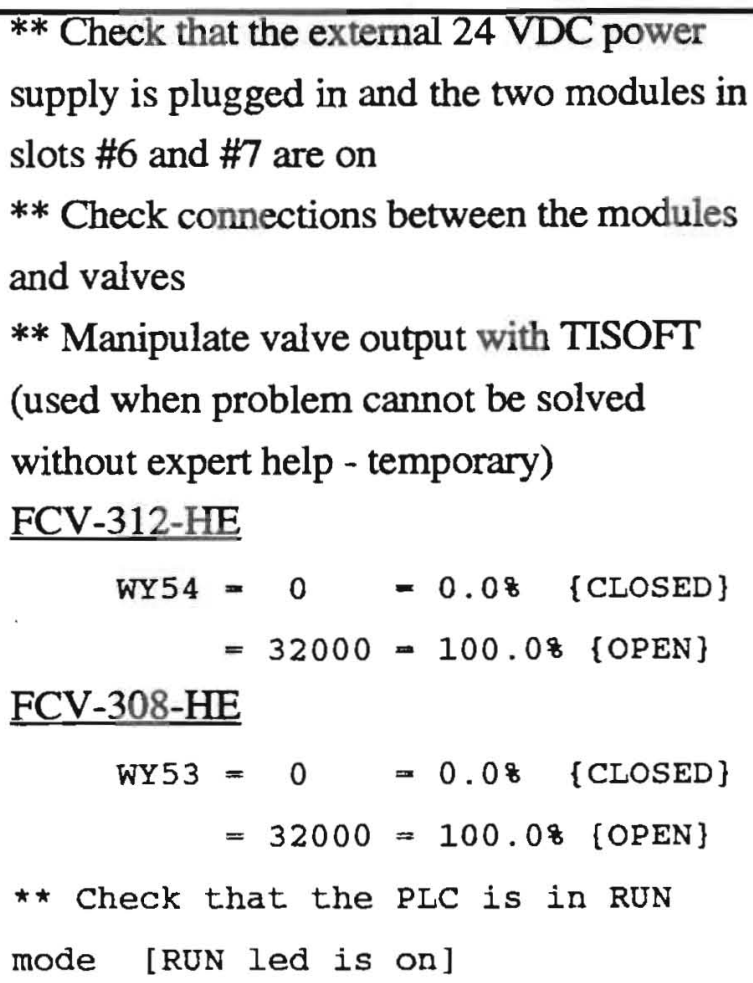 \\
\hline RTDs are reading incorrectly & $\begin{array}{l}\text { ** Check connections at modules and at } \\
\text { VLPC }\end{array}$ \\
\hline $\begin{array}{l}\text { Question marks on screen where data should } \\
\text { be present EX. ????? ohms }\end{array}$ & $\begin{array}{l}\text { ** Click on question mark and place tags } \\
\text { on-scan } \\
* * \text { Check that tag exists in the database }\end{array}$ \\
\hline
\end{tabular}




\section{References}

1. Siemens Programming Reference Manual

2. Intellution VO Driver Manual (TID)

3. Siemens Analog I/O Modules User Manual

4. CTI 2557 Isolated RTD Input Module User Manual

5. D0 Upgrade Fiber Tracker Detector 1024 Channel VLPC Cassette

Techincal Design Review, March 7th and 8th, 1996 
D0 TI555 I/O MAP (511)

16-3ul-97

\begin{tabular}{|c|c|c|c|c|c|c|c|c|c|}
\hline Channel & Base & SLOT/PT & I/O Type & Address & DMACS Tagname & Description & Terminal & Sex & Range \\
\hline 1 & 0 & $01 / 01$ & Analog In & WX0001 & PT619 & Pressure Transmitter for Helium sample sp & & & $0-25 \mathrm{psig}$ \\
\hline 1 & 0 & $01 / 02$ & Analog In & WX0002 & & & & & \\
\hline 1 & 0 & $01 / 03$ & Analog In & WX0003 & & & & & \\
\hline 1 & 0 & $01 / 04$ & Analog In & WX0004 & & & & & \\
\hline 1 & 0 & $01 / 05$ & Analog In & WX0005 & & & & & \\
\hline 1 & 0 & $01 / 06$ & Analog In & WX0006 & & & & & \\
\hline 1 & 0 & $01 / 07$ & Analog In & WX0007 & & & & & \\
\hline 1 & 0 & $01 / 08$ & Analog In & WX0008 & & & & & \\
\hline$\$$ & 0 & $04 / 01$ & RTD In & WX0025 & TE200-T & Temp. element on Cassette 1 & & & \\
\hline$\ell$ & 0 & $04 / 02$ & RTD In & WX0026 & TE202-T & Temp. element on Cassette 2 & & & \\
\hline 1 & 0 & $04 / 03$ & RTD In & WX0027 & TE204-T & Temp. element on Cassette 3 & & & \\
\hline 1 & 0 & $04 / 04$ & RTD In & WX0028 & TE206-T & Temp. element on Cassette 4 & & & \\
\hline l & 0 & $04 / 05$ & RTD In & WX0029 & TE304-T & Temp. element on liquid helium supply & & & \\
\hline 1 & $\mathbf{0}$ & $04 / 06$ & RTD In & WX0030 & TE306-T & Temp. element on LHe cold block return & & & \\
\hline 1 & 0 & $04 / 07$ & RTD In & WX0031 & TE310-T & Temp. element on GHe intercept return & & & \\
\hline 1 & 0 & $04 / 08$ & RTD In & WX0032 & L2 & & & & \\
\hline 1 & 0 & $04 / 09$ & RTD in & WX0033 & L4 & & & & \\
\hline 1 & $\mathbf{0}$ & $04 / 10$ & RTD in & WX0034 & L6 & & & & \\
\hline 1 & 0 & $04 / 11$ & RTD in & WX0035 & L8 8 & & & & \\
\hline 1 & 0 & $04 / 12$ & RTD in & WX0036 & M2 & & & & \\
\hline 1 & 0 & $04 / 13$ & RTD in & WX0037 & M4 & & & & \\
\hline 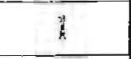 & 0 & $04 / 14$ & RTD in & WX0038 & M6 & & & & \\
\hline 1 & 0 & $04 / 15$ & RTD in & WX0039 & M8 & & & & \\
\hline$\overline{1}$ & 0 & $04 / 16$ & RTD in & WX0040 & & & & & \\
\hline 1 & 0 & $06 / 01$ & Analog Out & WY0049 & HTR200-1 & $\# 1 \& \# 2$ heater elements on Cassette 1 & & & \\
\hline 1 & 0 & $06 / 02$ & Analog Out & WY0050 & HTR202-3 & \#1 \& \#2 heater elements on Cassette 2 & & & \\
\hline 1 & 0 & $06 / 03$ & Analog Out & WY0051 & HTR204-5 & $\# 1 \&$ \& heater elements on Cassette 3 & & & \\
\hline 1 & 0 & $06 / 04$ & Analog Out & WY0052 & HTR206-7 & $\# 1$ \& \#2 heater elements on Cassette 4 & & . & \\
\hline 1 & 0 & $06 / 05$ & Analog Out & WY0053 & FCV308HE & Flow control valve for cold block He outlet & & & \\
\hline
\end{tabular}




\begin{tabular}{|c|c|c|c|c|c|c|c|c|c|}
\hline Channel & Base & SLOT/PT & I/O Type & Address & DMACS Tagname & Description & Terminal & Sex & Range \\
\hline 1 & 0 & $06 / 06$ & Analog Out & WY0054 & $\mathrm{FCV} 312 \mathrm{HE}$ & Flow control valve for $\mathrm{GHe}$ intercept $\mathrm{He}$ ou & & , & \\
\hline 1 & 0 & $06 / 07$ & Analog Out & WY0055 & & & & & \\
\hline 1 & 0 & $06 / 08$ & Analog Out & WY0056 & & & & & \\
\hline 1 & 0 & $07 / 01$ & Analog Out & WY0057 & & & & & \\
\hline 1 & 0 & $07 / 02$ & Analog Out & WY0058 & & & & & \\
\hline 1 & 0 & $07 / 04$ & Analog Out & WY0060 & & & & & \\
\hline 1 & 0 & $07 / 05$ & Analog Out & WY0061 & & . & & & \\
\hline 1 & 0 & $07 / 06$ & Analog Out & WY0062 & & & & & \\
\hline 1 & 0 & $07 / 07$ & Analog Out & WY0063 & & & & & \\
\hline 1 & 0 & $07 / 08$ & Analog Out & WY0064 & & & & & \\
\hline
\end{tabular}




\section{' ladder logic'}

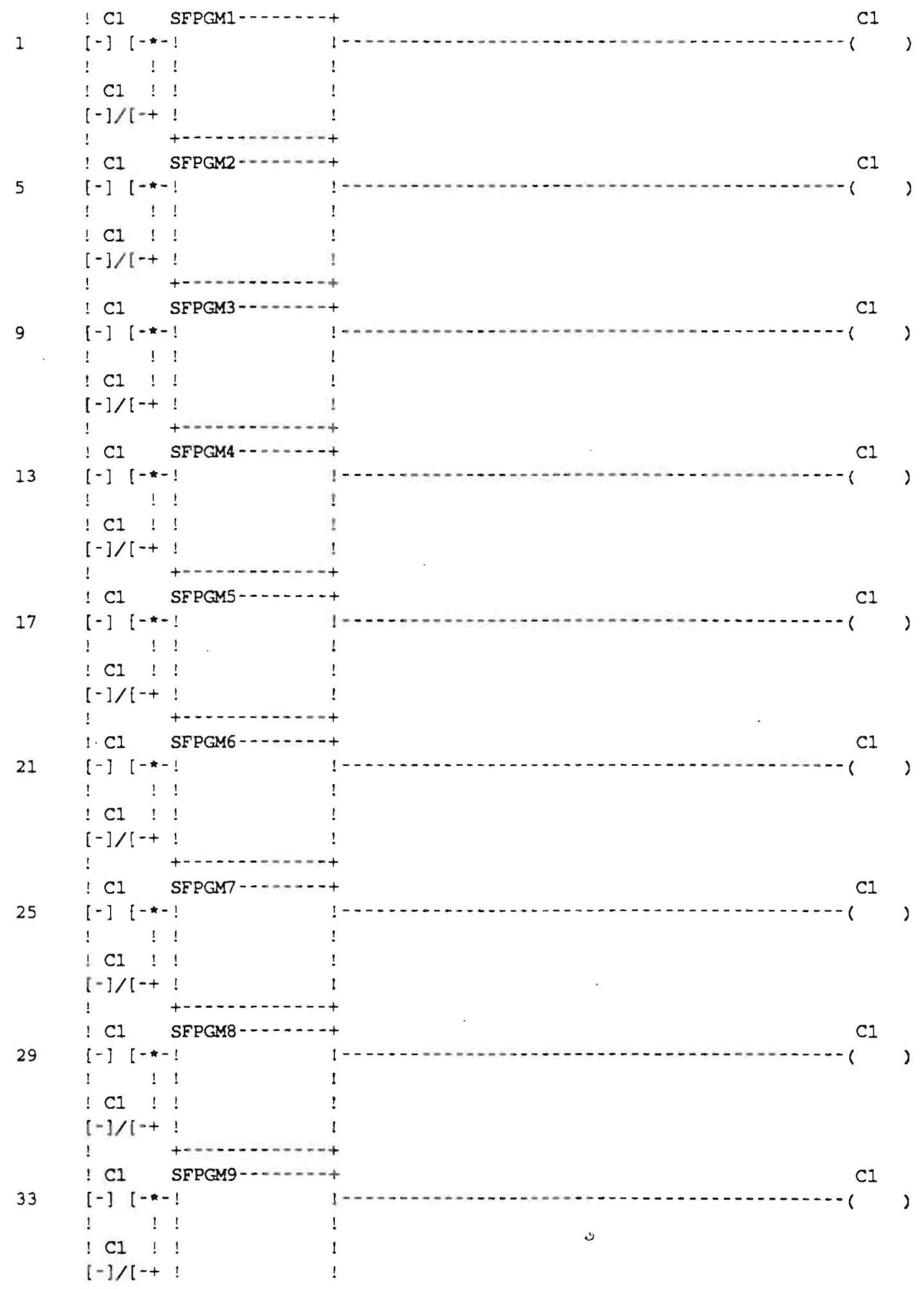




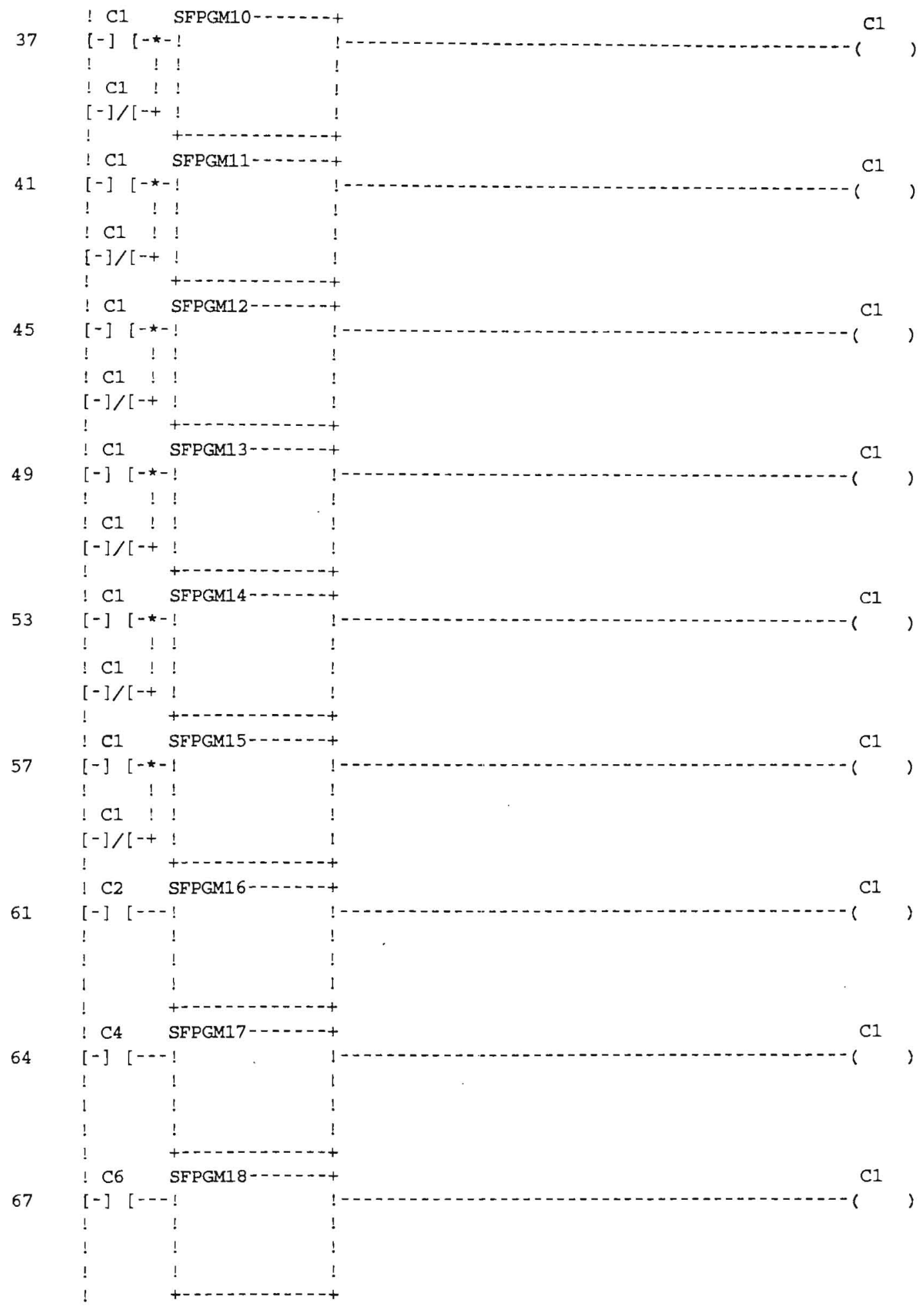




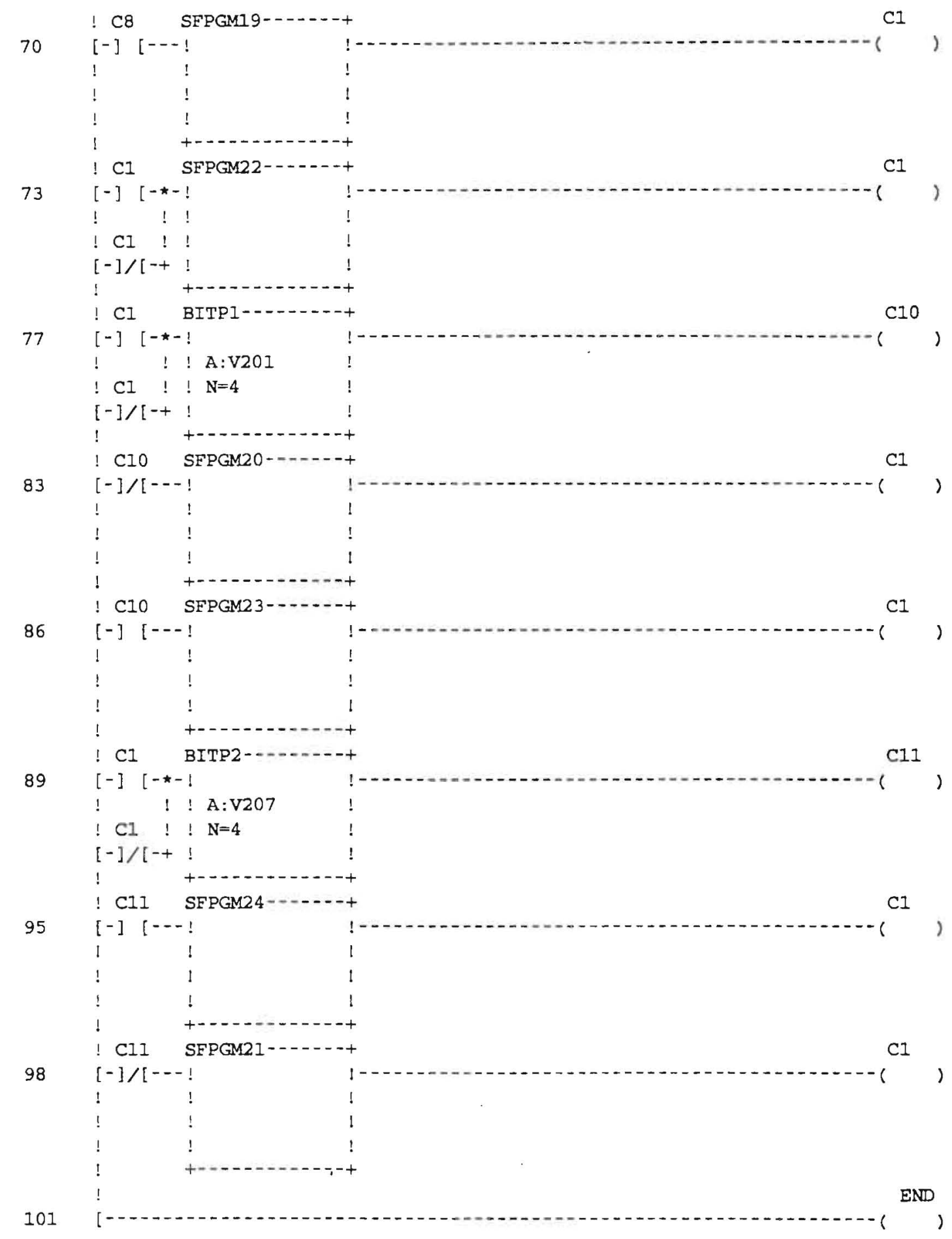




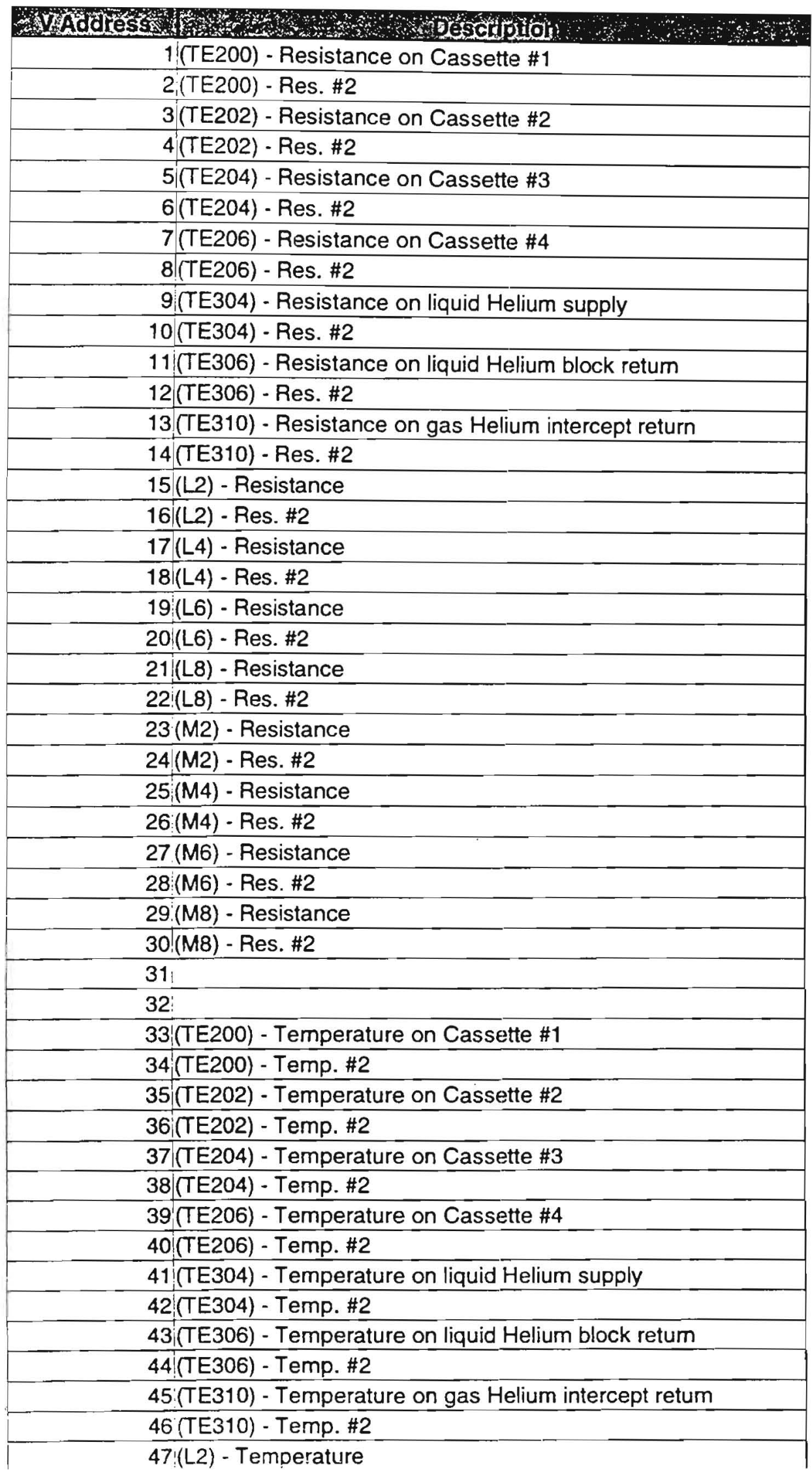




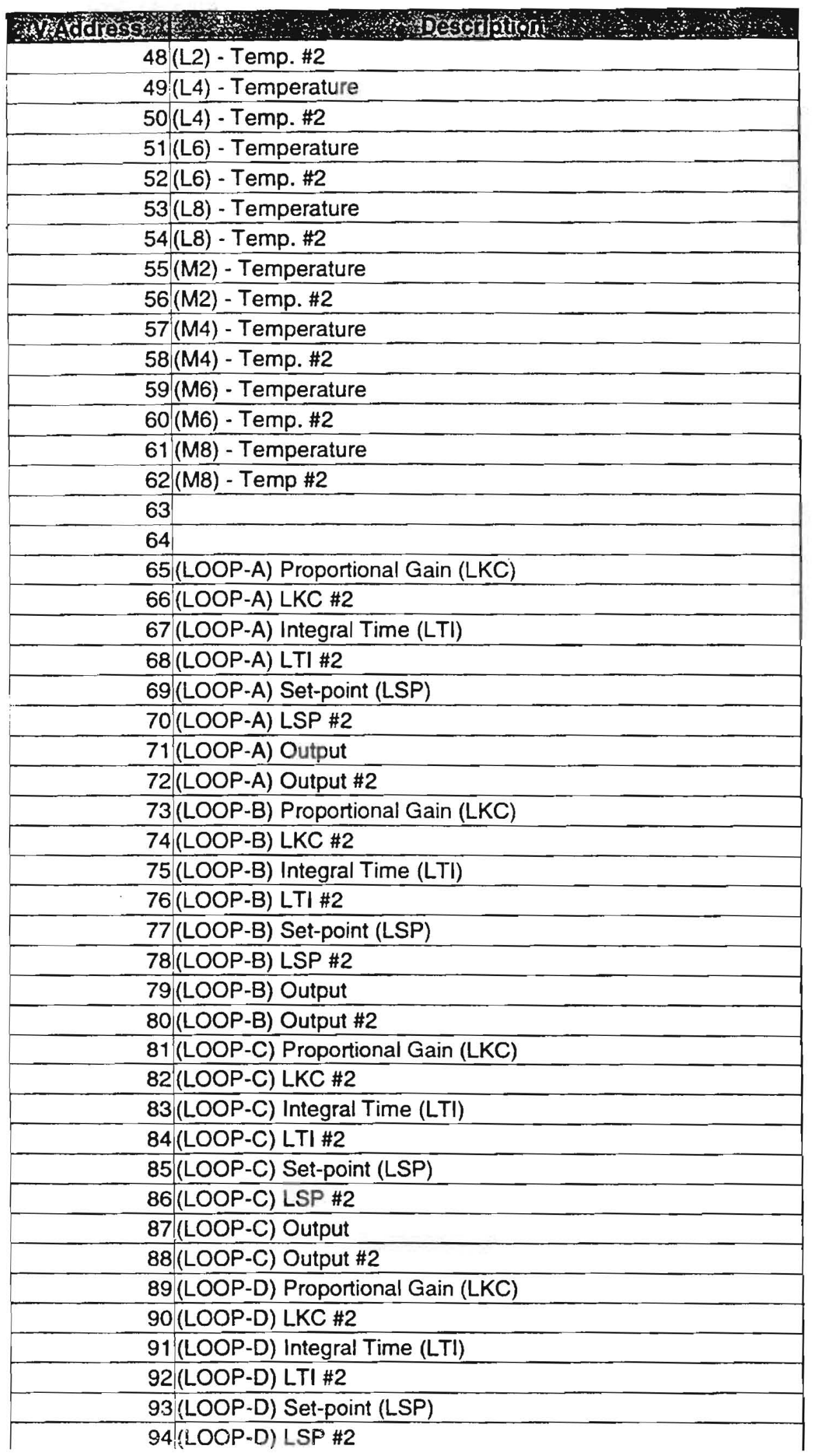




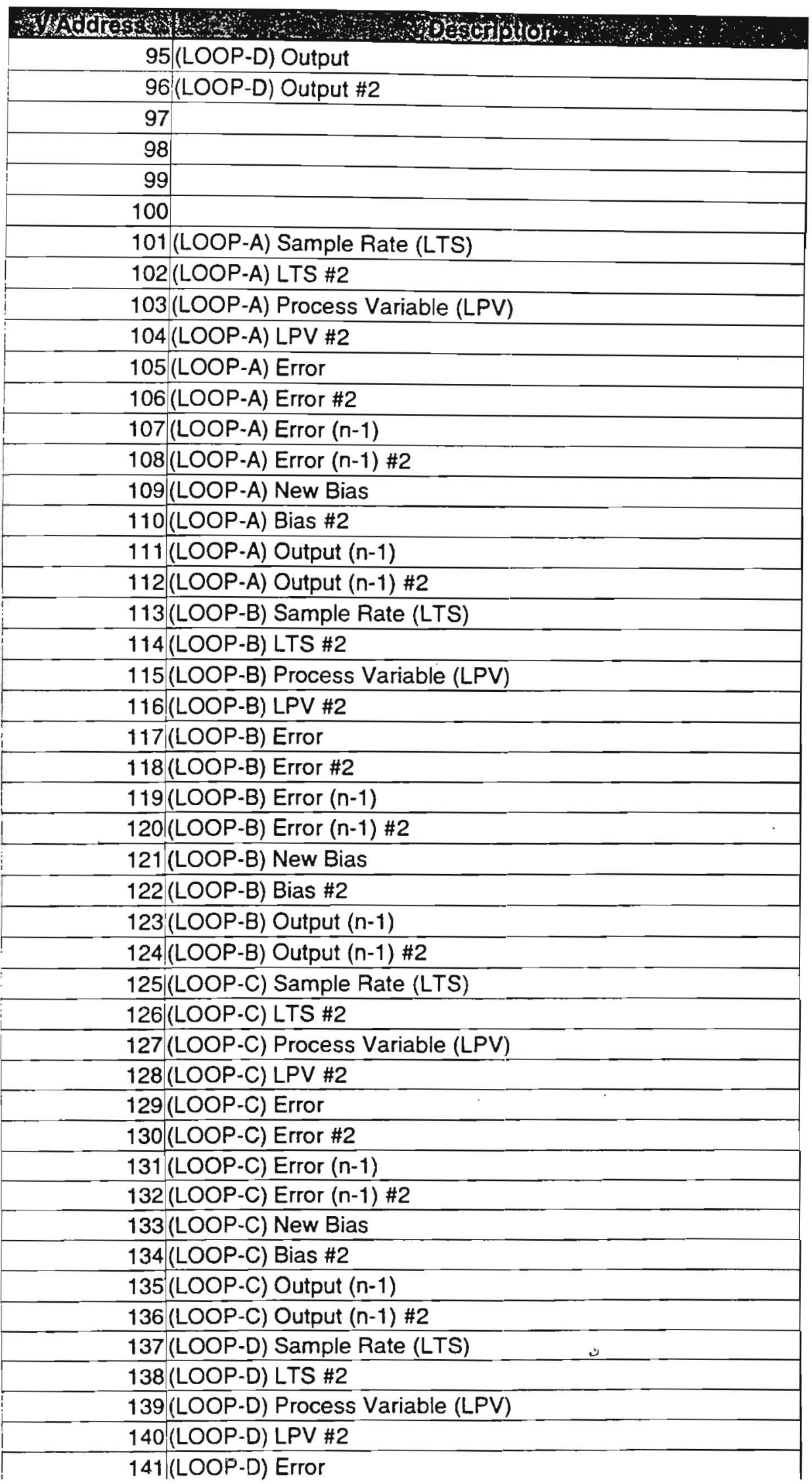




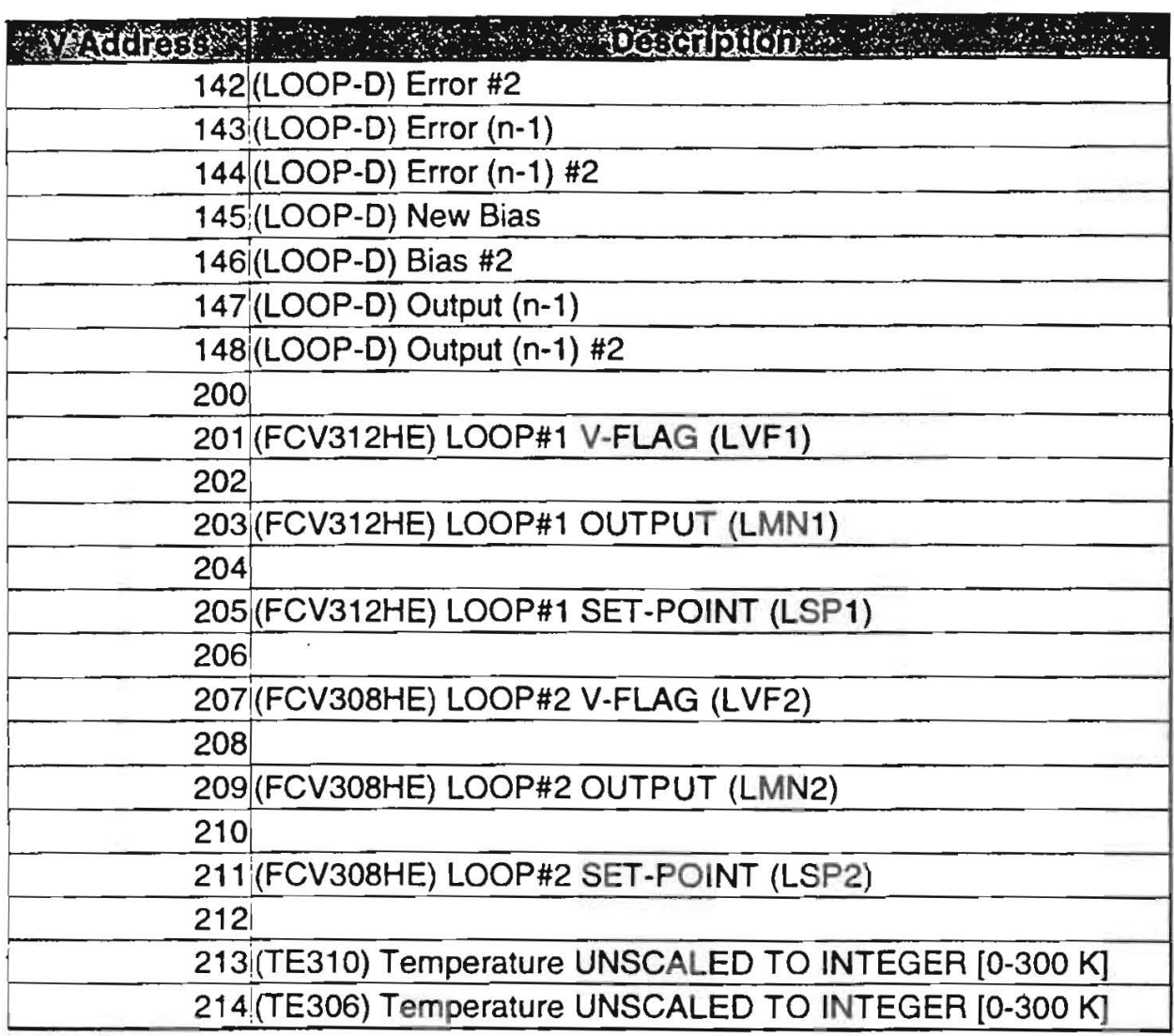




\begin{tabular}{|c|c|c|}
\hline Coil a & DB BMagnamo: & Qpentag \\
\hline 1DEFAULT & & \\
\hline 2 (LOOP-A) Manual / Automatic control & & \\
\hline 3 -unused- & & \\
\hline 4(LOOP-B) Manual / Automatic control & & \\
\hline 5 -unused- & & \\
\hline 6(LOOP-C) Manual / Automatic control & & \\
\hline 7 -unused- & & \\
\hline 8 (LOOP-D) Manual / Automatic control & & \\
\hline 9 -unused- & & \\
\hline 10 (FCV312HE) BIT COMPARE - Turn ON/OFF SFPGM & & \\
\hline 11 (FCV308HE) BIT COMPARE - TURn ON/OFF SFPGM & & \\
\hline 12 & & \\
\hline
\end{tabular}


505 I/O CONFIGURATION CHART FOR CHANNEL ... 1 BASE .... 00 I/O POINTS

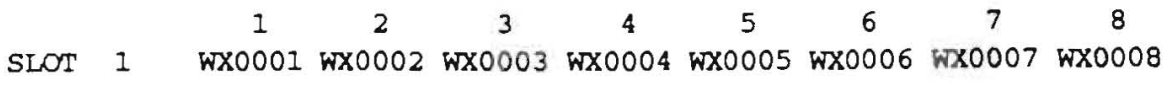

SLOT 2

SLOT 3

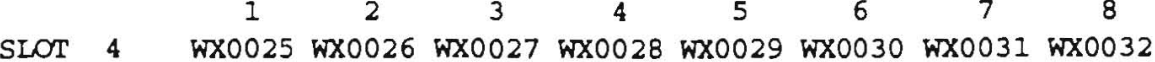

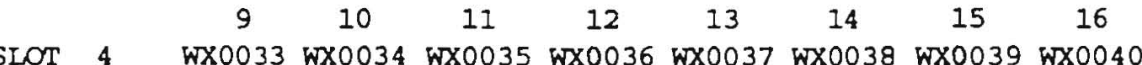

SLOT 5

SLOT 6 WY0049 WY0050 WY0051 WY0052 WY0053 WY0054 WY0055 WY0056

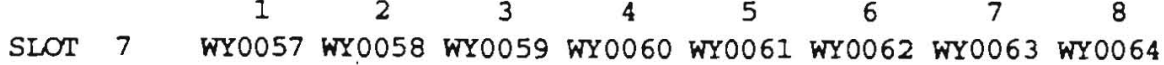

SLOT 8

SLOT 9

SLOT 10

SLOT 11

SLOT 12

SLOT 13

SLOT 14

SLOT 15

$\begin{array}{cccccccccc} & & 1 & 2 & 3 & 4 & 5 & 6 & 7 & 8 \\ \text { SF SLOT } & 16 & \text { WX016I } & \text { WX0162 } & \text { WY0163 } & \text { WY0164 } & \text { WY0165 } & \text { WY0166 } & \text { WY0167 } & \text { WY0I68 }\end{array}$ 


\section{SLOT \#1. \\ ANALOG INPUT MODULE \\ Appendix A Terminal Block Worksheet}

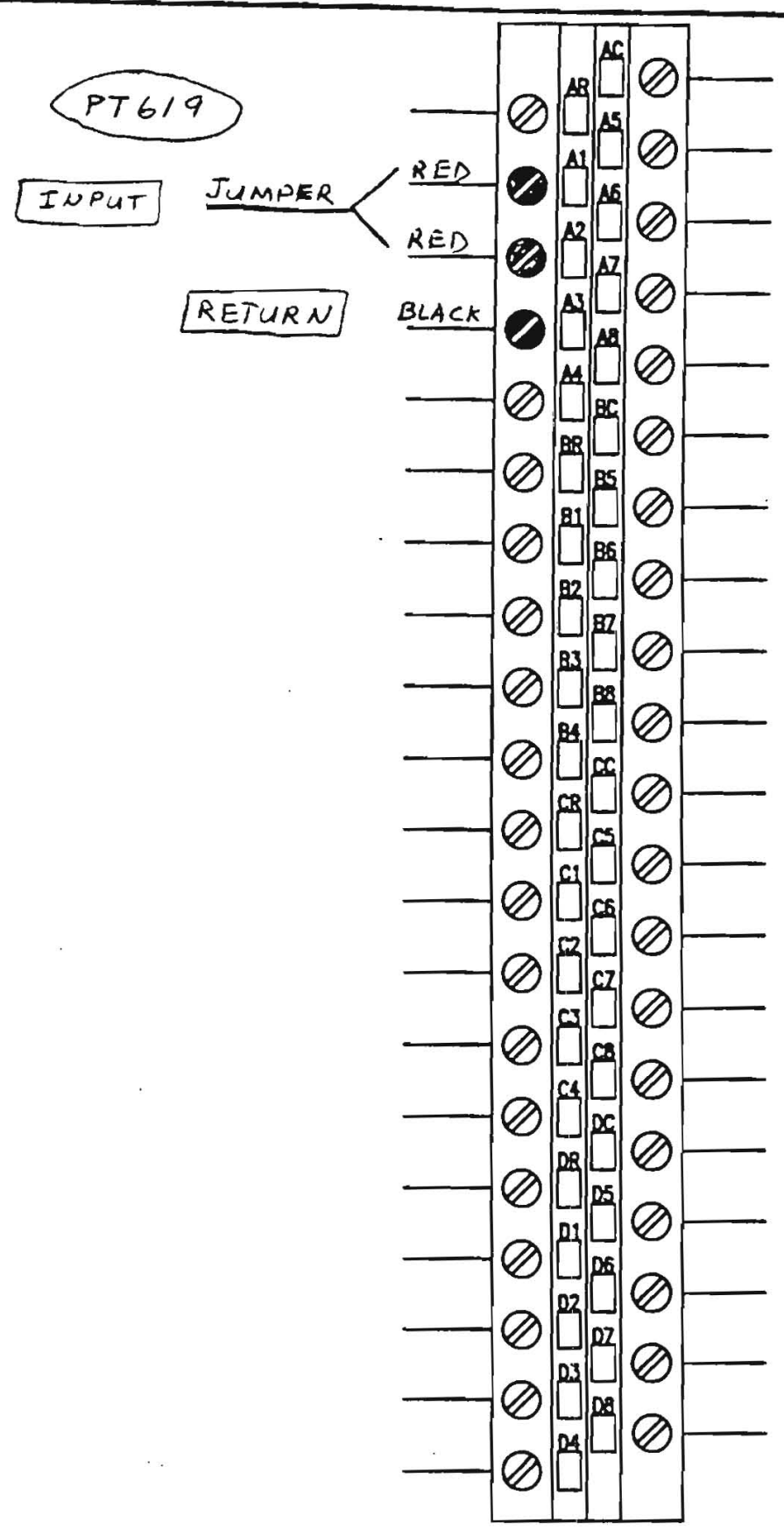




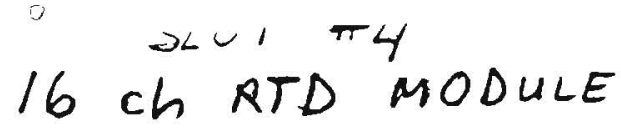

To assign an input to a specific channel, locate the appropriate channel position on the press in connector block as shown in the following figure (each channel consists of 4 positions).

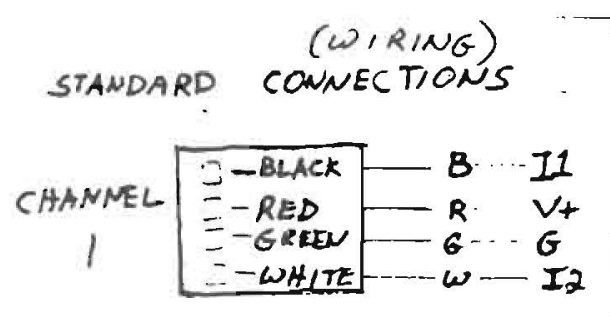

I1, Vt, G. I2

$B \quad R: G W$

( $L A C K)(R D)(G H E N)$ (WHTE)

$*$ signifies, different than
standard wiving.

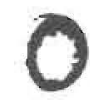

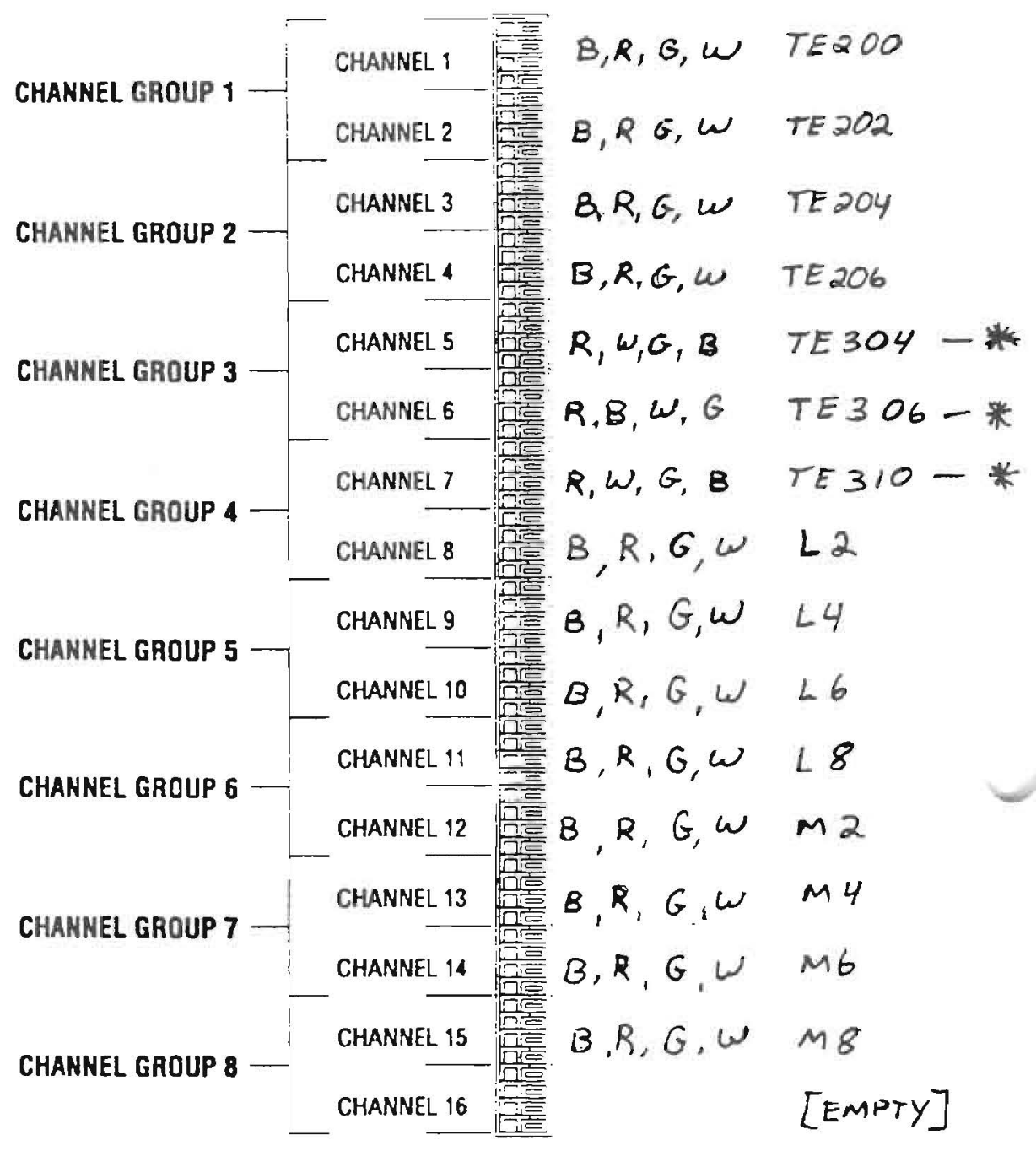

Figure 12 Press In Wiring Connector

\section{CAUTION:}

$R T D$ wires must be of the same gauge for proper lead length compensation. 


\section{SLOT \# 6 \\ ANALOG OUTPUT MODULE \\ Appendix A \\ Terminal Block Worksheet}

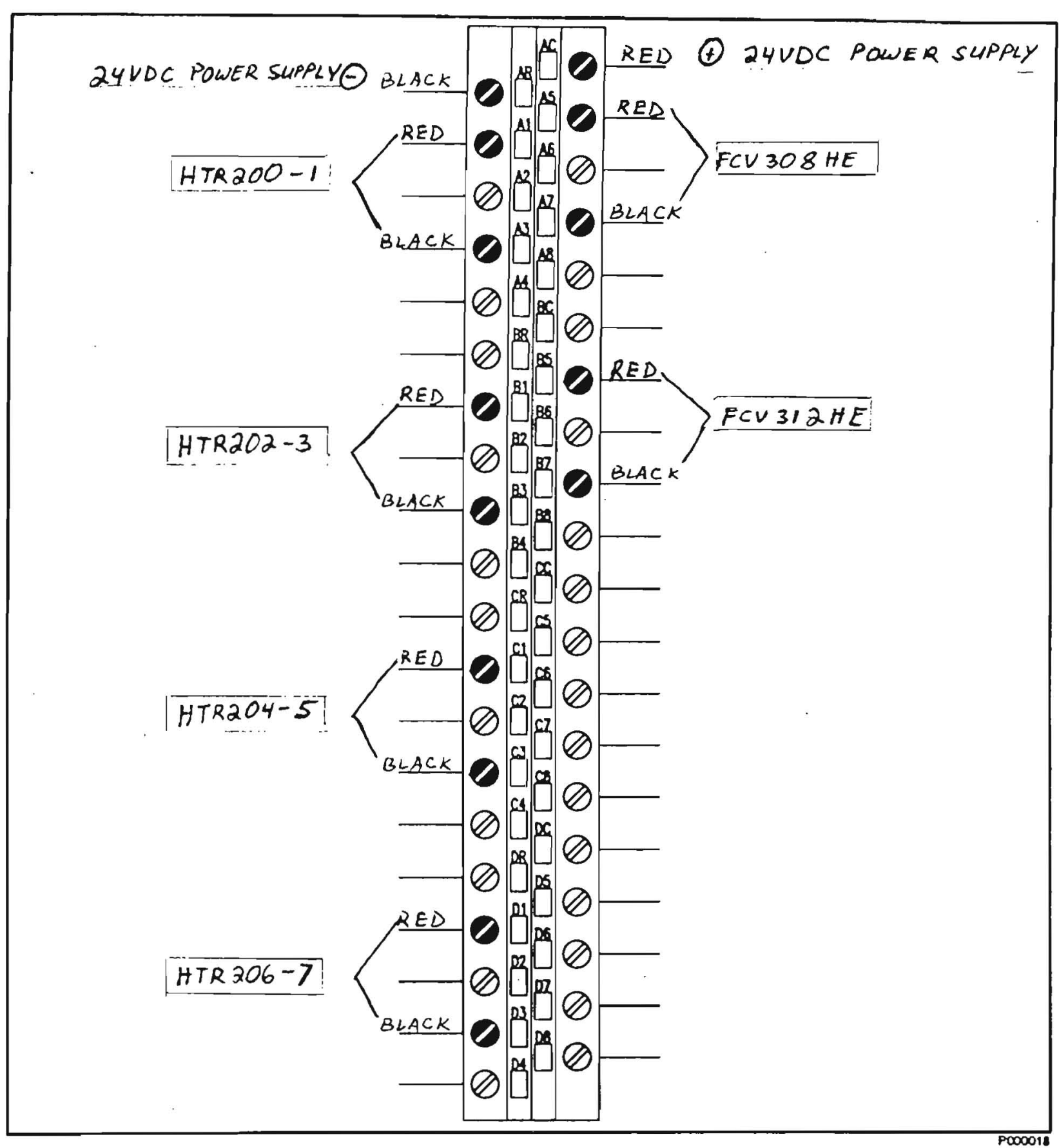


ERROR STATUS ADDR $(Y, C, W Y, V)$ :

PROGRAM TYPE (N,P,C,R): CYCLIC CYCLE TIME (SEC) : 3.0

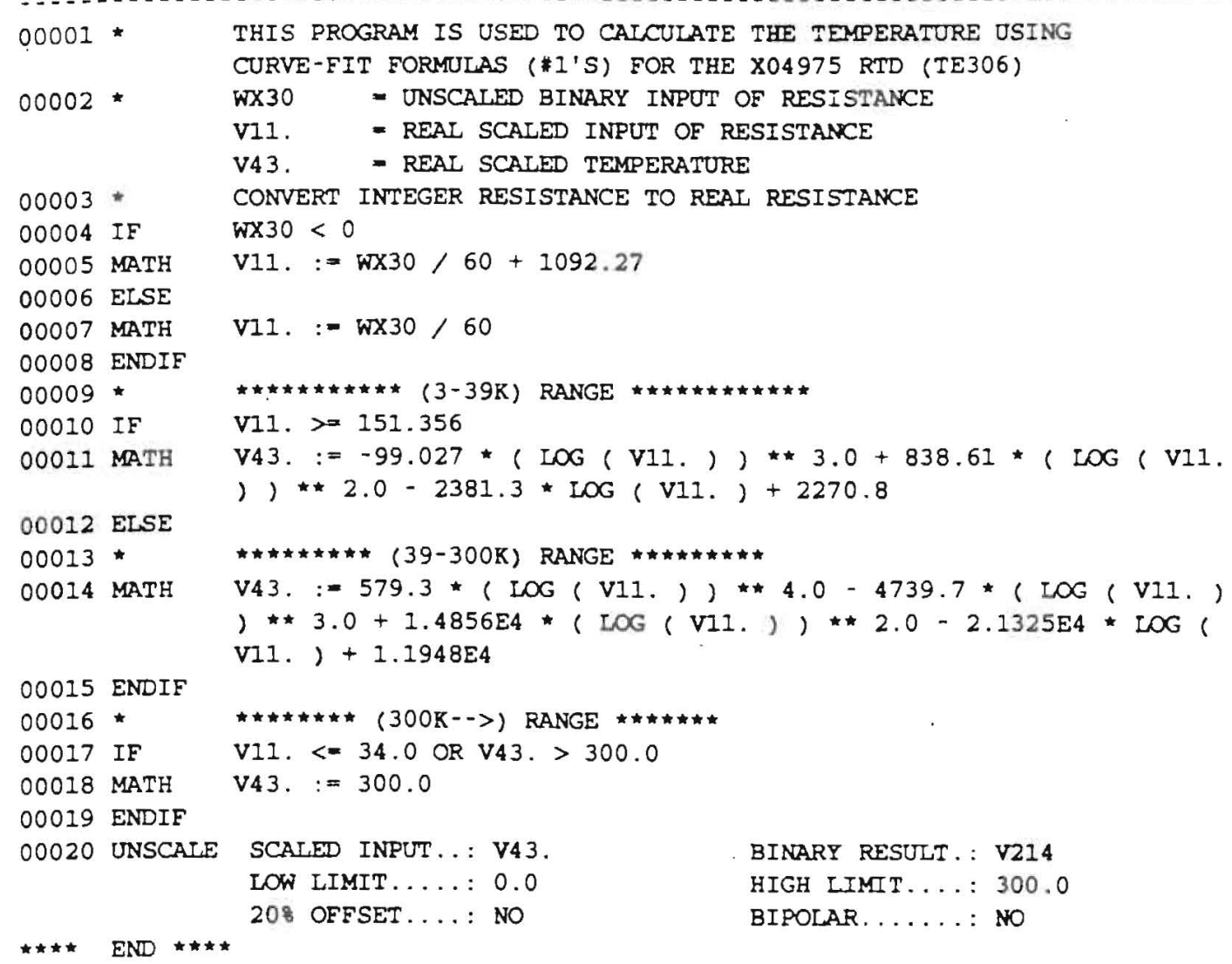




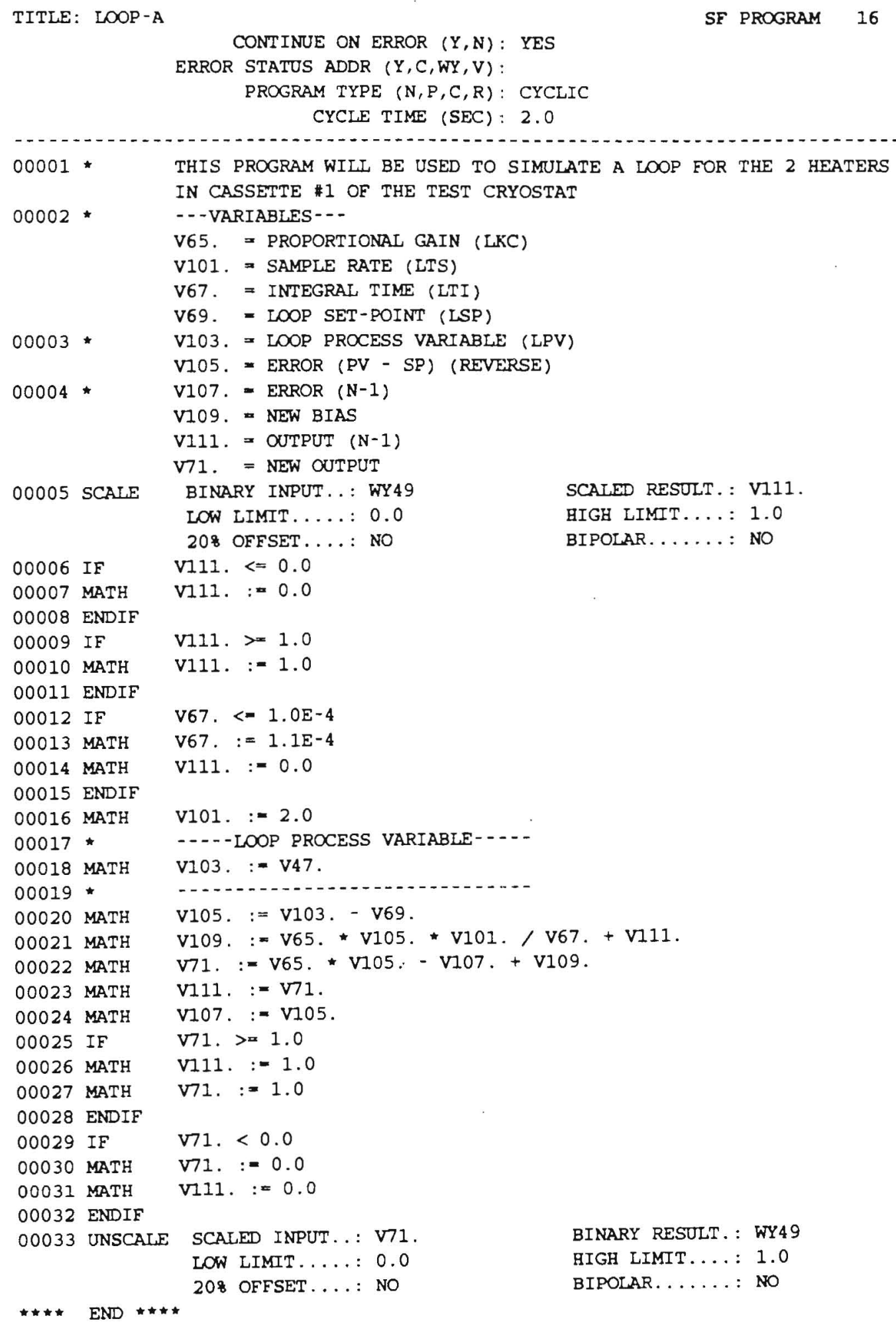




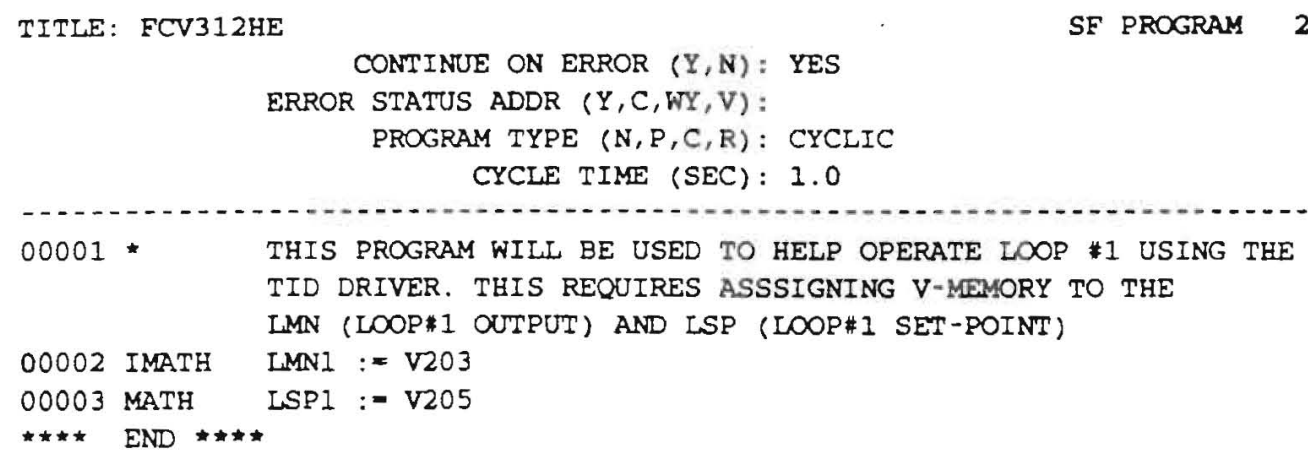


TITLE: STATUS1

CONTINUE ON ERROR $(Y, N)$ : YES

ERROR STATUS ADDR $(Y, C, W Y, V)$ :

PROGRAM TYPE (N,P,C,R): CYCLIC

CYCLE TIME (SEC): 0.5

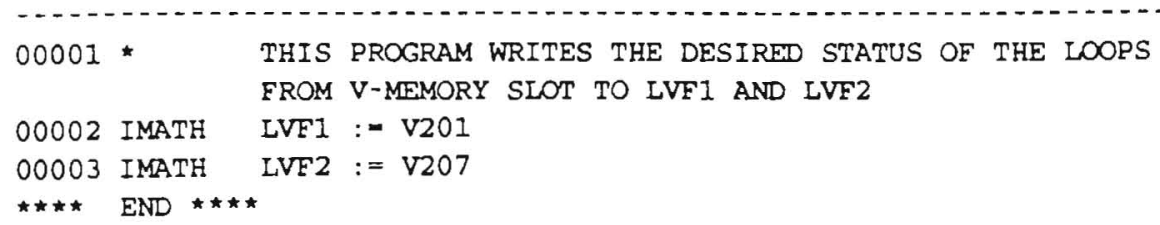


TITLE: 312-AUTO

ERROR STATUS ADDR ( $Y, C, W Y, V)$ :

PROGRAM TYPE (N,P,C,R): CYCLIC CYCLE TIME (SEC): 0.5

$\begin{array}{ll}00001 * & \text { THIS SPECIAL FUNCTION IS RUN WHEN THE LOOP } 1 \text { IS IN AUTOMATIC } \\ 00002 \text { IMATH } & \text { VODE. THE LOOP OUTPUT (IMNI) IS WRITTEN TO V-MEMORY V203 } \\ 0 & \text { LMNI }\end{array}$




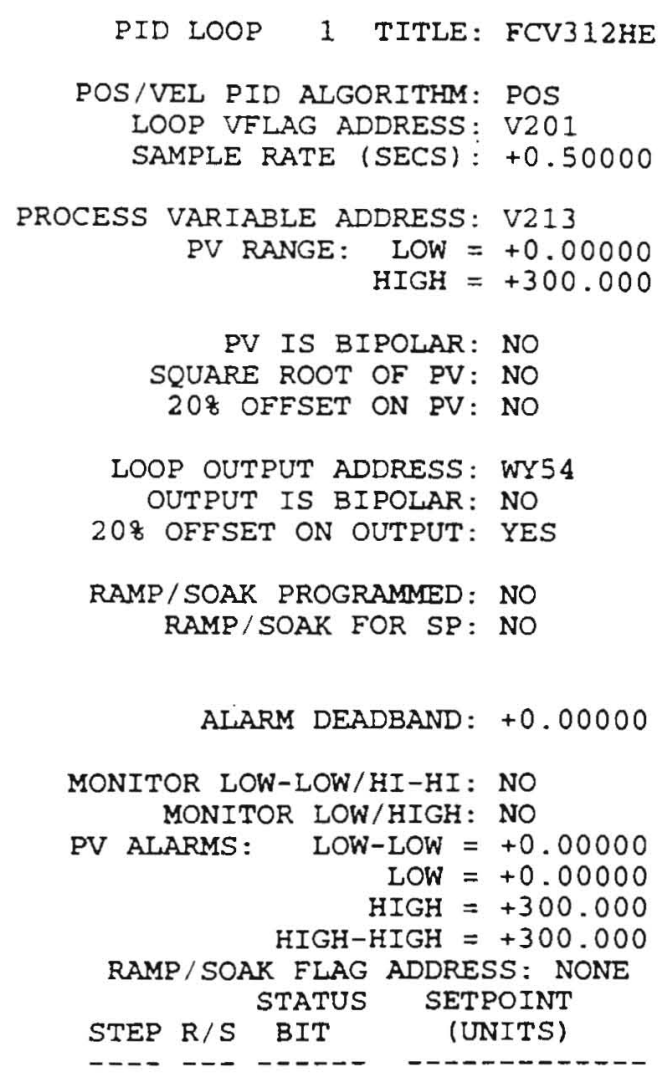

REMOTE SETPOINT: NONE

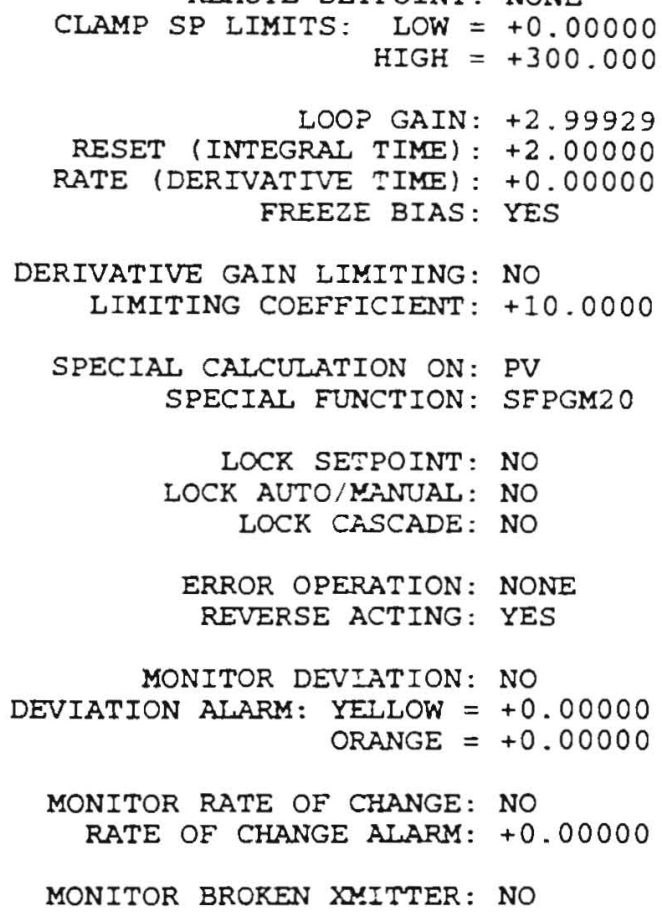




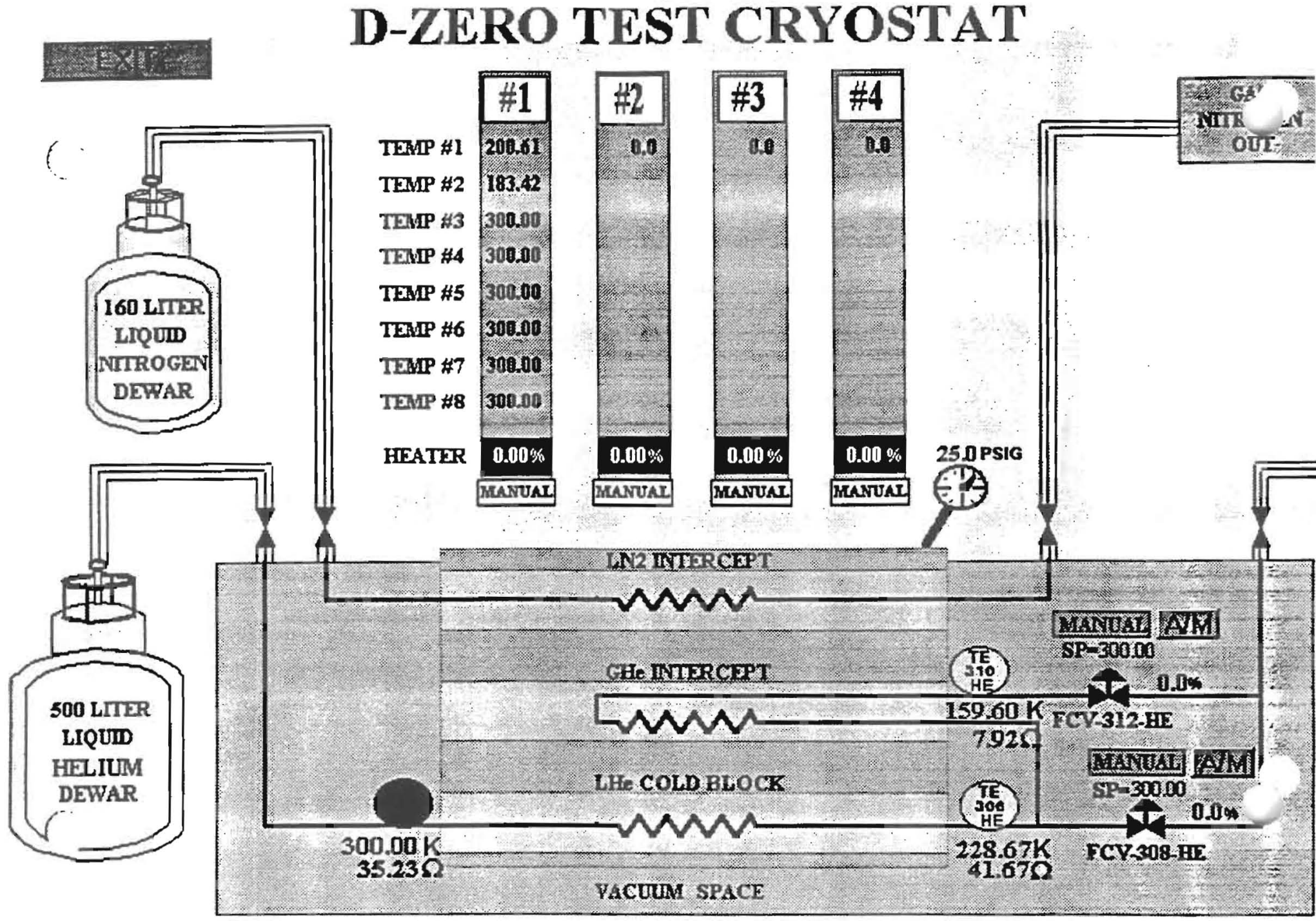




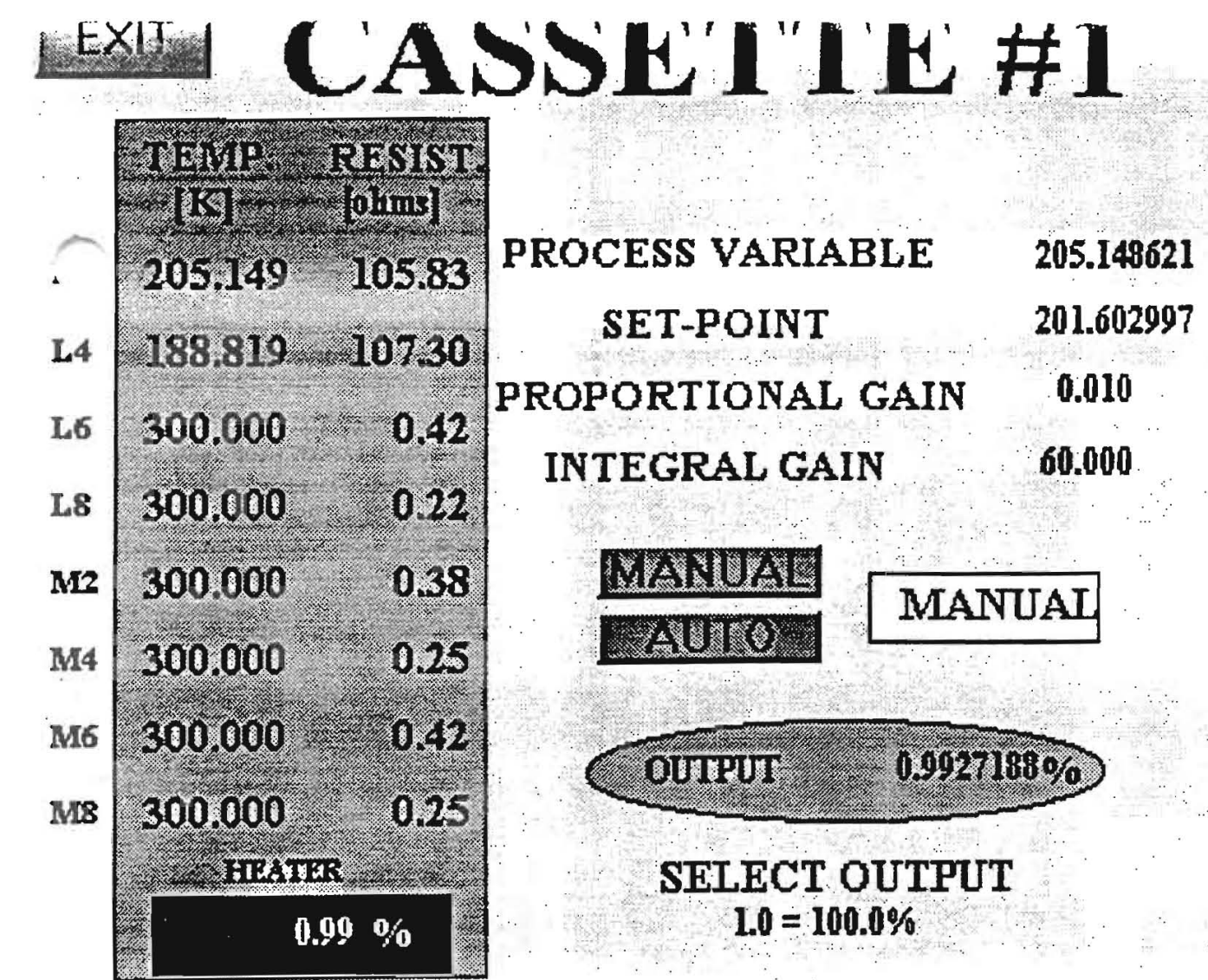




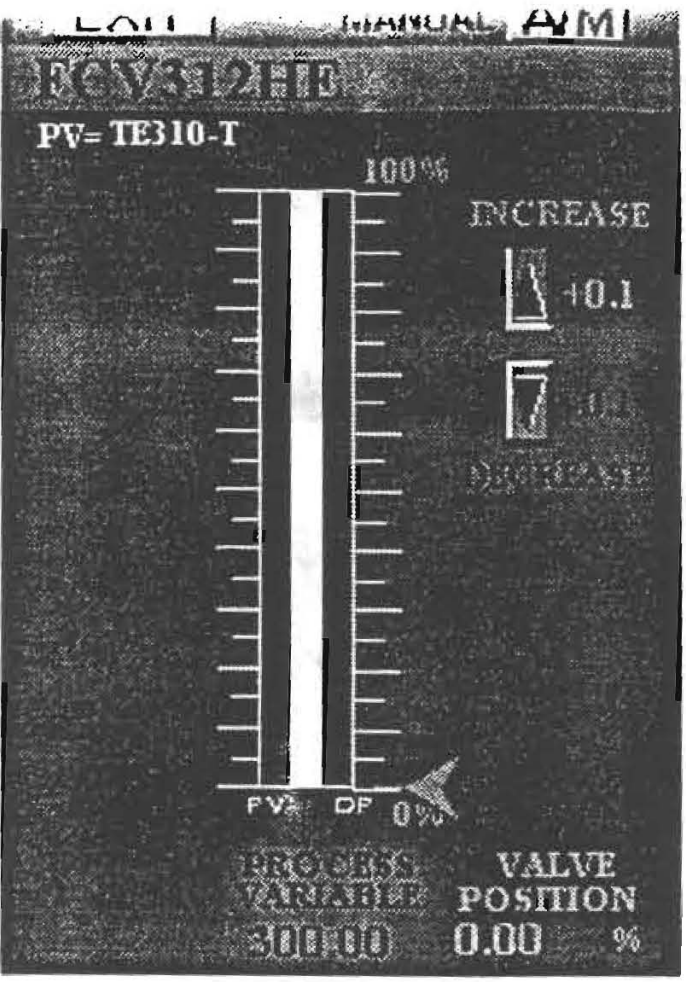


ADDENDUM TO:

D0 ENGINEERING NOTE: 3823.113-EN-470

Zaczek, Mariusz

8-27-97

D-Zero Mechanical Group 


\section{Purpose}

The purpose of this addendum is to list the new additions to the 3823.113-EN-470 engineering note for the test cryostat.

\section{Additions}

The new addition presented in this addendum deals solely with the loop programming of the valves FCV-312-HE and FCV-308-HE. A need has risen for the ability of the controller to change the loop gains -- proportional (P), integral (I) and derivative (D) gains. This has been achieved through the addition of special function program 25 (see the end of this addendum). Special function 25 compares two variable memory values and updates particular gains as necessary. As an example, let us analyze the programming of the proportional gain control for valve FCV-312-HE. In the DMACS environment variable memory V215. serves as both the input of the proportional gain and the output. The user input a desired gain (V215.) which is then sent to the PLC where the value is compared with V227. ( temporary $2^{\text {nd }}$ proportional gain). If V215. is less than or greater than V227. then this value is copied into LKC1. If the values are equal no update of LKC1 is performed. The next two lines constantly copy the value of LKC1 to V215. and V217.

The above special function thus allows the user to both input the desired gains while also viewing the current gain. If this function had not been employed, one might change a gain value in TISOFT, for example, and this would not be updated in DMACS, thereby possibly confusing the operator.

An additional ladder logic rung has been added to activate SFPGM25 (see below). 
Figure 1: SFPGM 25 Ladder Logic Rung

Also, more V-memory has been allocated to perform the necessary calculations:

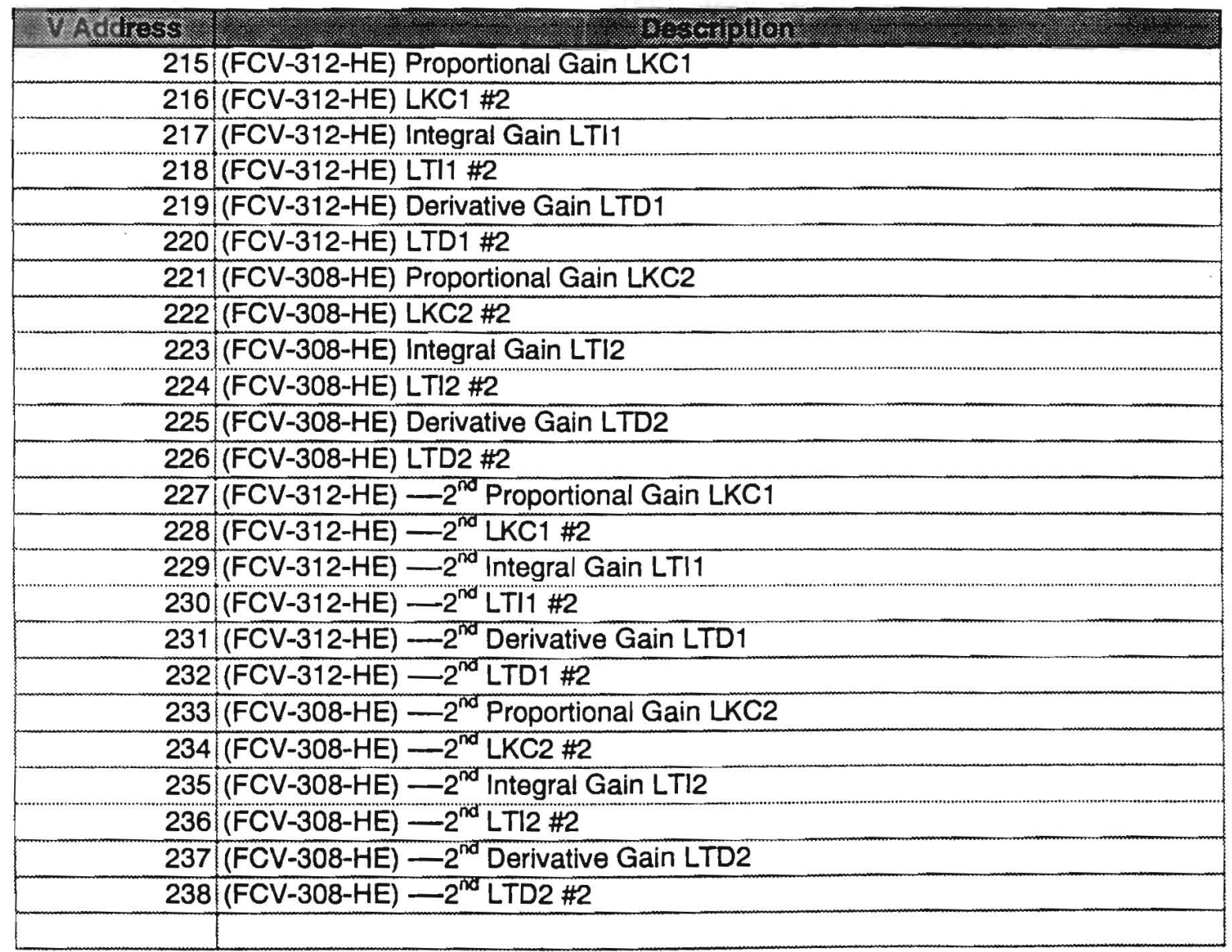




\section{VLV_GAIN (SFPGM25)}

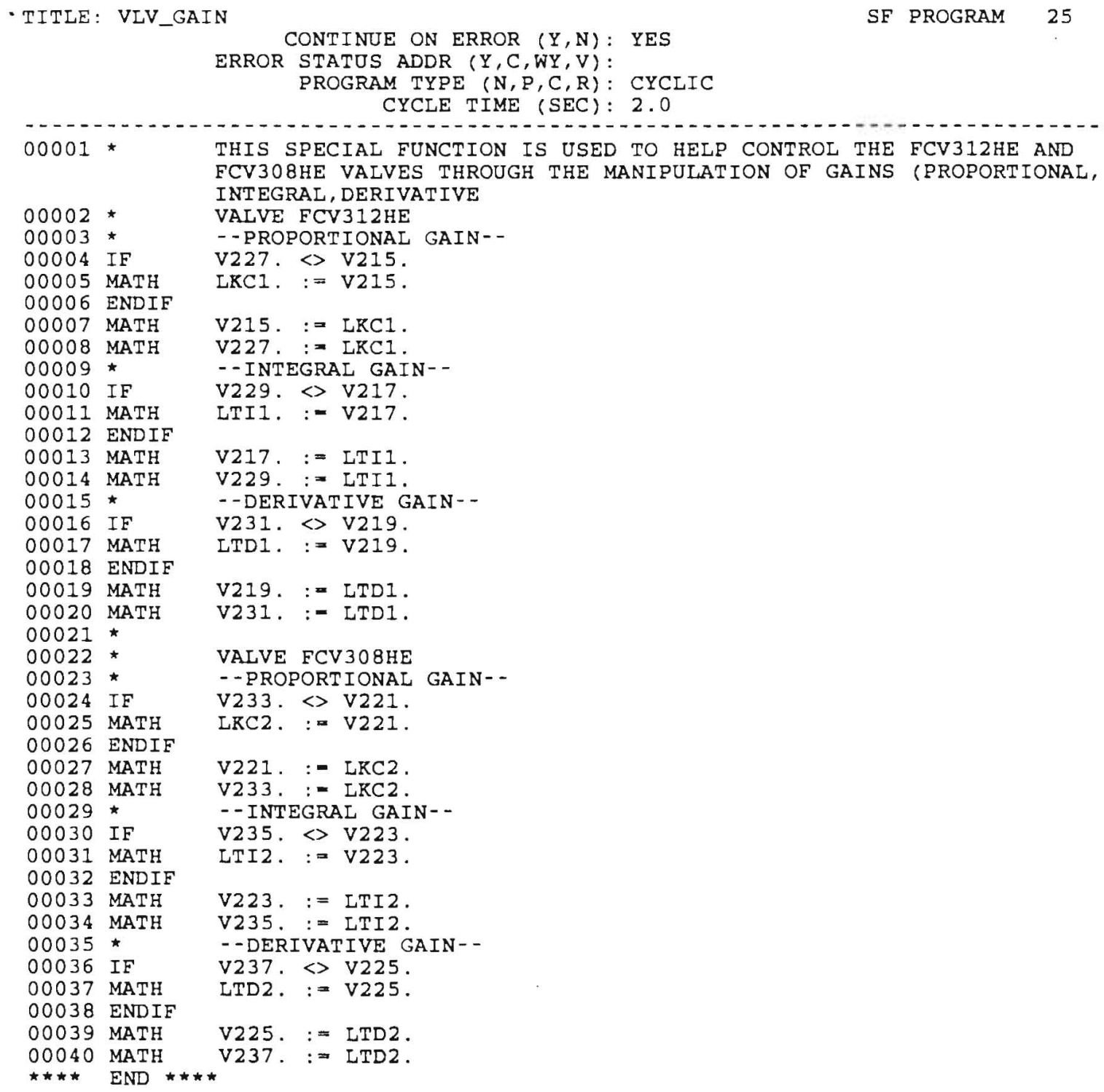

